\title{
Surface imaging with atomic and molecular features
}

\section{A new inversion technique and first numerical tests ${ }^{\star}$}

\author{
I. S. Savanov and K. G. Strassmeier
}

Astrophysical Institute Potsdam (AIP), An der Sternwarte 16, 14482 Potsdam, Germany

e-mail: [isavanov;kstrassmeier]@aip.de

Received 25 April 2005 / Accepted 11 August 2005

\begin{abstract}
We present a new Doppler-imaging inversion code that uses quasi-optimal filtering of the object's principal components of a Fisher information matrix. The new code allows one to perform the reconstruction of stellar surface temperature maps using molecular features like TiO, $\mathrm{CO}, \mathrm{OH}$, $\mathrm{CN}$ etc. which are numerous in spectra of late-type dwarfs. It is possible to use atomic and molecular features simultaneously in the restoration. Our tests verify the robustness of OPC but also emphasize its dependency on the various atomic and molecular line lists. Without inclusion of molecules, Doppler imaging of stars with photospheric temperatures below $\approx 4250 \mathrm{~K}$ (K7-M0) remains unreliable.
\end{abstract}

Key words. stars: imaging - stars: activity - methods: data analysis

\section{Introduction}

With the advent of 8-10 m-class telescopes and powerful new spectrographs, it is possible to extend Doppler-imaging to the cool (and faint) end of the main sequence. At a spectral type of $\approx \mathrm{M} 2$, stars are thought to become fully convective and cannot possess an overshoot layer between a radiative core and a convective envelope that, as in the case of the Sun and similar stars, likely harbors the dynamo. Therefore, one could expect a fundamentally different magnetic field topology than on the Sun and thus a qualitatively different surface temperature distribution with new, hitherto unknown, magnetic activity phenomena.

Unfortunately, atomic lines of cooler stars become progressively harder to observe because the thermal energy is too small to populate the higher atomic levels and also because more atoms are being bound in molecules and are missing from the atomic line formation. Stars cooler than M 2 can only be mapped by considering the many molecular bands, mostly TiO, $\mathrm{OH}$ and CO. Spectrum synthesis of the optical TiO bandheads at $705.5 \mathrm{~nm}$ and $886.0 \mathrm{~nm}$ by, e.g., O'Neal et al. (1998, 2005) already emphasized their sensitivity to surface temperature inhomogeneities. Furthermore, large starspots with temperatures up to $2000 \mathrm{~K}$ below the photospheric temperature will contribute a spectrum that is qualitatively different to the photospheric one and is partially dominated by molecules rather than atomic transitions (see, e.g., the NSO atlas of the

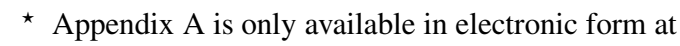
http://www.edpsciences.org umbral sunspot spectrum; e.g. Wallace et al. 1999). Therefore, one needs to include molecules in the Doppler imaging process of very cool stars (Berdyugina 2002).

The Doppler imaging technique is one of the most powerful tools for the investigation of stellar surface structure and its associated magnetic fields. Vogt et al. (1987) were the first to apply the inverse technique to high-resolution spectra of cool stars. Numerous subsequent contributions were made by many authors, see the review by, e.g., Collier Cameron (2001). At present, different surface imaging techniques have been applied to $\approx 65$ active late-type stars with the total number of Doppler images reaching 245 (Strassmeier 2002 and references therein). The discovery of polar starspots and anti-solar differential surface rotation was among the surprising results.

Currently, Doppler imaging procedures differ mostly by the applied minimization techniques and the various inputs for the line profile calculations. A recent review of codes developed for these purposes, including magnetic Doppler imaging, can be found in Rice (2002). Most of the unpolarized-light image restorations of active stars are made with four codes; the TEMPMAP code (e.g. Rice \& Strassmeier 2000) employing either the maximum entropy method or the Tikhonov method as the imaging technique, the code by Collier Cameron (1992) and Donati \& Brown (1997), both also employing the maximum entropy method, and the code by Berdyugina (1998) involving the Occamian approach and the mean information principle. Among these codes only the one by Berdyugina (1998) includes calculation of opacities and intensities of atomic and molecular lines. 
In the this paper, a new approach based on the analysis of eigenvectors of the Fisher information matrix filtered with a nonlinear filter of the Kolmogorov-Wiener type is applied to the surface imaging problem. Our new code, the OPC (object principal components) inversion code, makes it possible to reconstruct temperature maps using local line profiles with molecular features from $\mathrm{TiO}, \mathrm{CO}, \mathrm{OH}, \mathrm{CN}$ etc. which are quite numerous in spectra of late-type stars. We study the robustness and limitations of the new code, apply it to artificial data with atomic and molecular features and carry out a large number of numerical experiments.

\section{Description of the code}

\subsection{The inverse problem with OPC}

Recently, Terebizh (2004) proposed a completely new way of constructing a nonlinear filter similar to the optimal Kolmogorov-Wiener filter within the statistical approach to inverse problems. According to this approach the linear Dopplerimaging transformation is a matrix problem of the form

$y_{0}=H x_{0}+\xi$,

where the $n \times 1$-vector $x_{0}$ is an unknown object (in our case the distribution of the $n$ parameters over the stellar surface), the $m \times n$-matrix $H$ is the point spread function (PSF), $\xi$ is random noise, and the $m \times 1$-vector $y_{0}$ is the observed image (in our case a set of profiles of a spectral line observed throughout the rotational period with $m$ data points). In the statistical approach to the inverse problems by Terebizh (1995) the unknown solution $x$ is considered as a statistical estimate of the object $x_{0}$, given its image, a PSF and the properties of the noise. The presentation of the linear form of the Dopplerimaging problem was discussed in numerous papers (e.g. Vogt et al. 1987; Collier Cameron 1992; Donati \& Brown 1997; Berdyugina 1998; Collier Cameron 2001).

In order to reduce the number $m$ of dimension of the vector that describes the misfit between the observed image and its estimate to number $n$, a simple procedure based on singular value decomposition is used, i.e., $H=U \Delta V^{T}$ (Press et al. 1992). We can define a vector $p_{0}$ of the object principal components

$p_{0}=V^{T} x_{0}$.

With

$\phi=U^{T} y_{0}$

we then obtain the final representation of the linear model:

$$
\phi=\Delta p_{0} .
$$

The advantages of the use of the refined image $\phi$ of length $n$ are especially appreciable when $m \gg n$. Like the familiar Fourier coefficients the principal components are often easier to recover than the object itself.

We can also define the least square estimate $x_{\star}$ of the unknown object and its principal components $p_{\star}$ (Terbizh 2004)

$x_{\star}=V p_{\star}, p_{\star}=\Delta^{-1} \phi$.
By introducing the appropriate set of weights $w_{k}$, which constitutes $W=\operatorname{diag}\left(w_{k}\right)$, into Eq. (5) we obtain

$x_{\mathrm{w}}=V W p_{\star}$.

The squared error of the filtered estimate $\left\langle\left\|x_{\mathrm{w}}-x_{0}\right\|^{2}\right\rangle$ can be minimized by the set of weights

$w_{\mathrm{k}}=\frac{\lambda_{\mathrm{k}} p_{0}^{2}}{\left(1+\lambda_{\mathrm{k}} p_{0}^{2}\right)}, \lambda=\Delta^{2}$

which constitutes the optimal Wiener filter.

The solution $p$ of the system

$$
\begin{aligned}
& \left\|\left[W(p)-E_{\mathrm{n}}\right] \phi\right\|^{2}<t_{1-\alpha}^{n} \\
& \left\|W(p) p_{\star}-p\right\|^{2}=\min
\end{aligned}
$$

allow us then to find the quasi-optimal estimates of the object and its principal components ( $\alpha$ is the adopted significance level for the reconstruction, $t_{\mathrm{q}}$ is the quantile of order $q$ ).

With the quasi-optimal filtering procedure we can reduce the influence of the small eigenvalues of the Fisher matrix

$I=H^{T} H$,

which defines the "feasible region" consisting of all the object estimates that have images with appropriate deviation from the observed image $y_{0}$ in the statistical sense. It is important that usually only a part of the principal components completely contain the information about the unknown object $x_{0}$. The ratio of the largest to the smallest eigenvalues of the Fisher matrix $I$ is many orders for typical inverse problems (in our case it is near $10^{8}-10^{10}$, which points out the strong instability of the problem). The idea of the objects principal components is not a new one (e.g. Hotelling 1933). Since that time many implementations of the principal components approach have been published and can be found in the current literature.

Further details of the approach can be found in the original papers by Terebizh $(1995,2004)$. Our consideration of the Fisher informational matrix gives us the possibility to estimate the errors of the solution and to obtain an indication which features of the image are significant.

\subsection{Local line profiles}

The astrophysical input includes a set of synthetic spectra at various aspect angles. The temperature range is usually $3500-6000 \mathrm{~K}$ and synthetic spectra are computed for nine angular points on the stellar disk. The radiative-transfer equations are solved with atmospheric models from the grid ap00k2odfnew (Kurucz 2000) using the spectrum codes of Tsymbal (1996) and Gray (2000). Both codes compute the electron number density and the number densities of important species, their ions and the relevant diatomic molecules. Specifically, the code of Gray (2000) solves a system of 7 non-linear equilibrium equations to determine the number densities and then proceeds with detailed opacity and optical depth computations. For further technical details see to the Spectrum V2.66 homepage ${ }^{1}$. The input list of atomic

\footnotetext{
${ }^{1}$ http://www.phys.appstate.edu/spectrum/spectrum.html
} 
parameters was obtained from the VALD database (Piskunov et al. 1995; Kupka et al. 1999). We use different inputs to the molecular line list, as was discussed in detail in Savanov (2005b). It includes the SCAN line list for the CH and CN red systems, Kurucz's $\mathrm{CDs}$ for the violet system $\mathrm{CN}$ and $\mathrm{OH}$, $\mathrm{NH}, \mathrm{SiO}, \mathrm{SiH}, \mathrm{MgH}, \mathrm{C}_{2}$ and $\mathrm{CO}$, and the data by Plez (1998) for TiO. Figure 1 compares a number of synthetic spectra used in our inversions and provides a rough estimate of the influence of parameters like effective temperature, gravity, microturbulence and chemical abundances for the reconstructions. A more detailed discussion follows in Appendix A.

\subsection{Improvements and limitations}

Instead of only atomic lines, as in codes like TEMPMAP and INVERS7 (see Piskunov \& Rice 1993), our input line list consists additionally to several hundreds of atomic lines of thousands of molecular lines (for a wavelength coverage of only $\approx 50 \AA$ as for the tests in this paper). All of these lines are used in the inversion simultaneously. Note that the code can be expanded to an arbitrary number of wavelength regions and thereby combines, e.g., particular infrared $\mathrm{OH}$ and $\mathrm{CO}$ bands and optical $\mathrm{TiO}$ bandheads with specific optical regions containing major atomic lines. Limits are solely set by the completeness (and reliability) of the line lists invoked and by the computational power available.

In principle, the simultaneous treatment of atomic and molecular species can lead to an unstable solution due to the different sensitivities of the solution to changes in temperature or input parameters in the presence of noise. One must also keep in mind the different origins of spectral details associated with atomic and molecular features. While most of the atomic lines originate from the entire stellar surface, its "bumps" are produced only by spots with temperatures lower than the quiet photosphere. At the same time, the TiO bands are formed solely in compact low-temperature spots. Another crucial difference between atomic and molecular species is connected to the fact that the molecular component of a single spatial element (pixel) on the stellar surface contributes to a broad spectral range whereas an atomic line contributes just inside its intrinsic (thermal and turbulent broadened) line profile.

No photometric input is currently possible. Results based on inversions of observed light curves will be discussed in a separate paper (Savanov \& Strassmeier, in prep.).

Our approach has the advantage, as does the Occamian approach of Terebizh \& Biryukov (1994) as exploited by Berdyugina (1998), that the eigenvectors of the Fisher matrix define the principal axes of the error ellipsoid in image space and allow an estimate of the error bars of the reconstructed image.

\section{Numerical simulations}

\subsection{Forward computations and assumptions}

The forward computations in this paper are done in a selfconsisted manner with the same routines that are used in the corresponding parts of the inverse code.
We consider two hypothetical K2V stars for the robustness tests of various line-profile reconstructions and another hypothetical, cooler, $\approx \mathrm{K} 7 / \mathrm{M} 0$ star to show the improvements by including molecules. The relevant stellar parameters for these test stars are listed in Table 1. For each case, we compute spectral line profiles from the test image of the stars in Fig. 2. Twenty equidistant rotational phases are considered. The product of all wavelength points at all rotational phases, a total number of over $\approx 5000$ data points for each set, comprises the onedimensional image vector $y_{0}$ in Eq. (1). We then compare the results from the inversion with the original image from Fig. 2 and tabulate the results in the appendix in Table A.1.

The number of excitation and vibrational transitions makes it impossible to test the impact of detailed changes that one could introduce into the line list, e.g., altering transition probabilities for each of the atomic lines or the wavelengths for the many molecular lines. Such changes are not considered in the tests in this paper. Before the application to real stellar data the line lists must be verified by comparing the synthetic spectra to high-quality observation of the Sun and other standard stars. This has been common practice, see e.g. Valenti et al. (1998), Berdyugina et al. (1998), Strassmeier et al. (1999), Hackman et al. (2001). However, once molecular line lists are involved, we need more than just one standard star with known atmospheric parameters and chemical abundances to cover the full temperature range between $3500 \mathrm{~K}$ to $6000 \mathrm{~K}$.

In all our tests a grid of $6^{\circ} \times 6^{\circ}$ cells on the stellar surface was used. With the adopted values of the inclination of the rotational axis, the visible stellar image consists of $n=1500 \mathrm{el}-$ ements for Star 1 and 3 and $n=1200$ elements for Star 2. We have adopted a surface grid of $15^{\circ} \times 15^{\circ}$ as well as $5^{\circ} \times 5^{\circ}$ for two test runs with $\mathrm{S} 1$ and found no basic differences in the restorations other than the lowered and increased resolution, respectively. CPU time is prohibitively longer for any other (higher) surface resolution.

\subsection{With or without molecules?}

Figure 3 is an example of a reconstruction when we ignored any molecular contribution. The forward computation was done with the full atomic and molecular line list, as in all the following tests, but the reconstruction ignored all molecules together (their line intensities were simply set to zero). For case 1 (Fig. 3a) OPC reconstructed the correct spot temperature but made the spot shape rather elongated and fuzzy. The spot size at $T_{\text {spot }}=3500 \mathrm{~K}$ decreased by $10 \%$ for the equatorial spots and by over $50 \%$ for the high-latitude spot. On the contrary, the spot size at $4000 \mathrm{~K}$ increased by $\approx 20 \%$ for all latitudes. Additionally, the polar regions are recovered with a temperature cooler by $\approx 200 \mathrm{~K}$ than the input model. Warmer regions of up to $\Delta T \approx 200 \mathrm{~K}$ appeared in between the cool spots at roughly the sub-observer latitude. These changes are due to the fact that the code has to compensate for the missing blanketing due to the many molecular lines.

For the low-inclination case, Star 2 (Fig. 3b), it is easier for OPC to reconstruct the true spot size-temperature ratio but it still underestimated the true spot model. The ideal 
a) Gravity
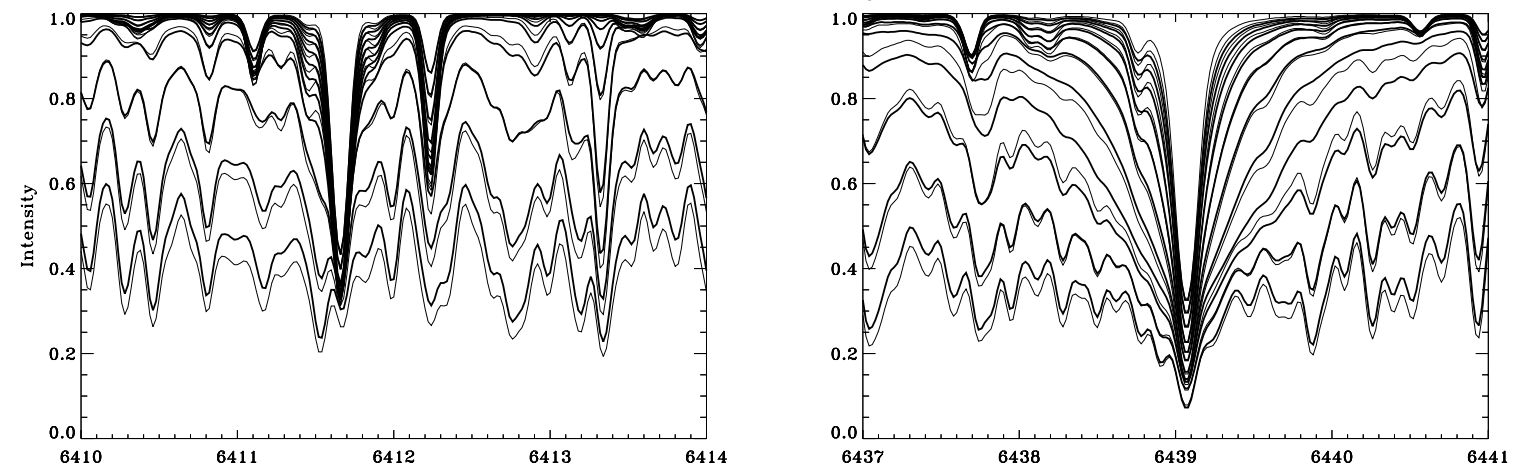

b) Microturbulence
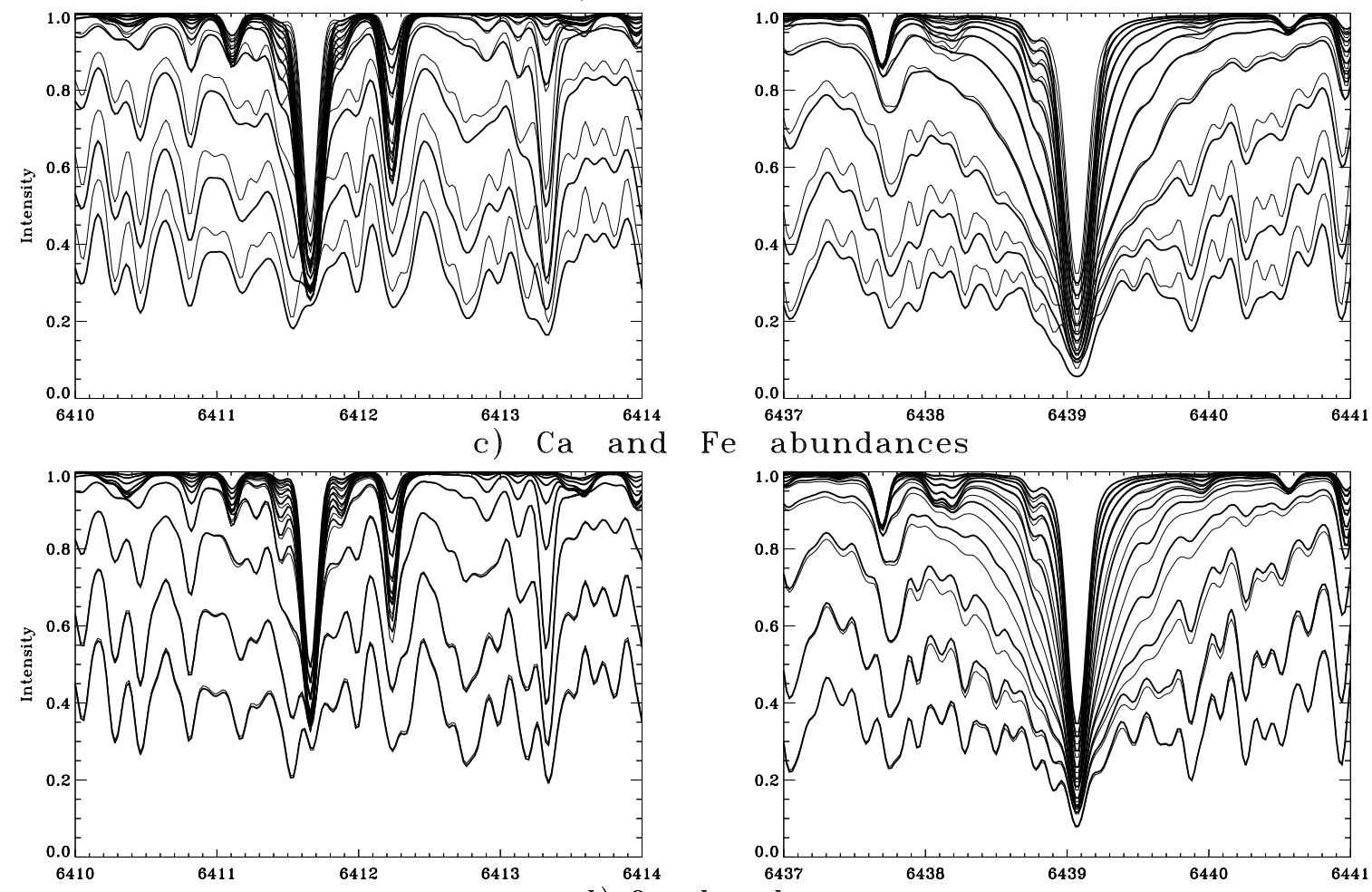

Fe abundances

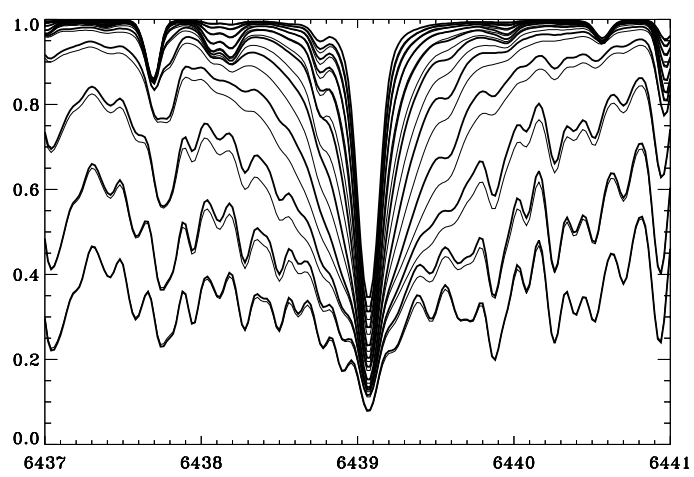

d) 0 abundance
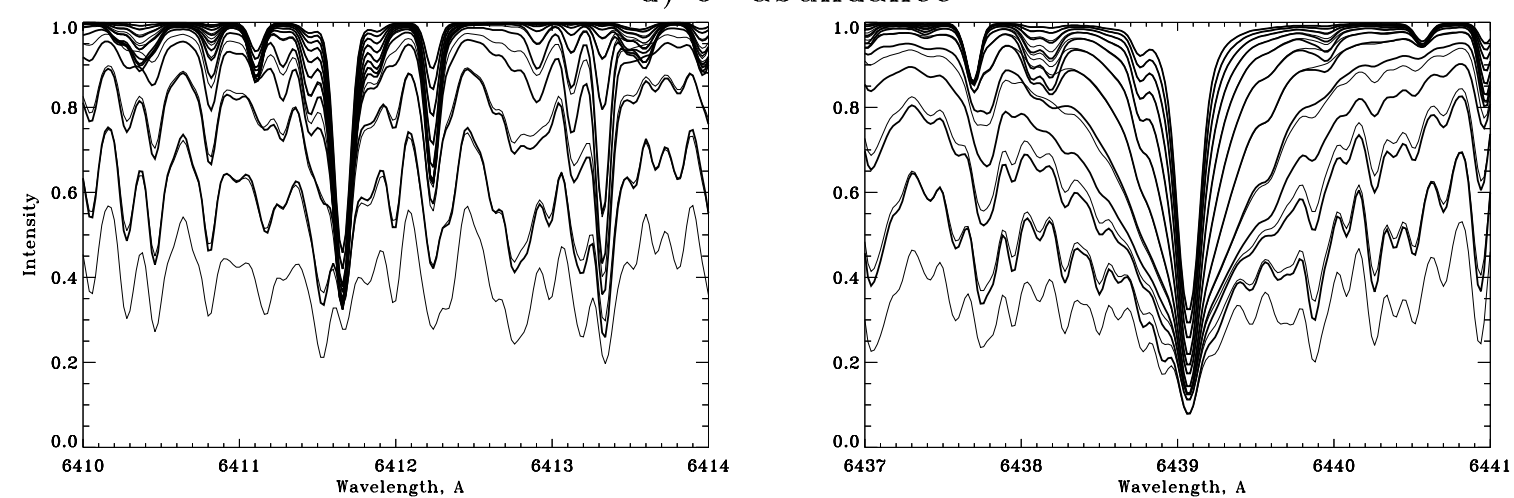

Fig. 1. Synthetic spectra for two wavelength regions containing the Fe I 6411-Å line (left column) and the Ca I 6439-Å line (right column). Each panel shows spectra for 11 values of $T_{\text {phot }}$ from 3500 to $6000 \mathrm{~K}$ from bottom to top (thin full lines). Gravity was set to log $g=3.5$. These spectra were computed at zero aspect angle and with zero microturbulence, the classical values for the damping constants, and were convolved with an instrumental profile corresponding to a spectral resolution of $R=60000$. Each thick line in the figure shows the synthetic spectrum altering one astrophysical parameter at a time, i.e., a) with gravity changed by $0.5 \mathrm{dex}$ to $\log g=4.0, \mathbf{b})$ microturbulence changed to $2.0 \mathrm{~km} \mathrm{~s}{ }^{-1}$, c) abundances of $\mathrm{Ca}$ and $\mathrm{Fe}$ reduced by 0.2 dex and, d), abundance of oxygen reduced by 0.2 dex. 
Table 1. Adopted parameters for the forward computations. $\mathrm{S} 1$ and S2 simulate roughly a K2V star, S3 a K7/M0V.

\begin{tabular}{|c|c|c|c|}
\hline Parameter & $\begin{array}{l}\text { Star } 1 \\
(\mathrm{~S} 1)\end{array}$ & $\begin{array}{l}\text { Star } 2 \\
(\mathrm{~S} 2)\end{array}$ & $\begin{array}{l}\text { Star } 3 \\
(\mathrm{~S} 3)\end{array}$ \\
\hline$T_{\text {phot }}, \mathrm{K}$ & 5000 & 5000 & 4250 \\
\hline$T_{\text {spot }}, \mathrm{K}$ & 3500 & 3500 & 3500 \\
\hline spot $\operatorname{area}^{1}, \%$ & 3.8 & 3.8 & 7.8 \\
\hline $\log g$ & 4.0 & 4.0 & 4.5 \\
\hline$v \sin i, \mathrm{~km} \mathrm{~s}^{-1}$ & 24 & 50 & 24 \\
\hline Inclination & $60^{\circ}$ & $30^{\circ}$ & $60^{\circ}$ \\
\hline Microturbulence, $\mathrm{km} \mathrm{s}^{-1}$ & 0.0 & 0.0 & 0.0 \\
\hline Macroturbulence, $\mathrm{km} \mathrm{s}^{-1}$ & 1.0 & 1.0 & 1.0 \\
\hline Abundance & solar & solar & solar \\
\hline
\end{tabular}

${ }^{1}$ In $\%$ of the entire sphere.

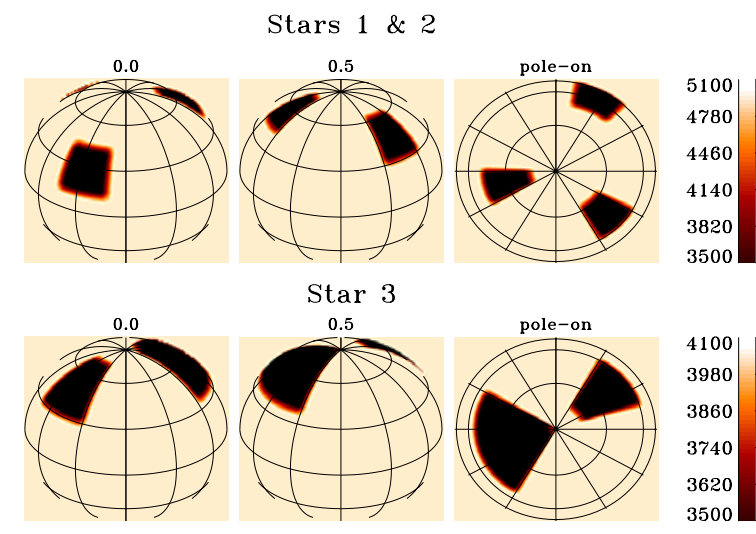

Fig. 2. The artificial test stars from Table 1. Stars 1 and 2 have three equally large rectangular spots centered at latitudes of $30^{\circ}, 45^{\circ}$ and $60^{\circ}$ and longitudes of $115^{\circ}, 225^{\circ}$ and $330^{\circ}$, respectively. Star 3 has two spots of different sizes centered at latitudes of $50^{\circ}$ and $60^{\circ}$ and longitudes of $50^{\circ}$ and $256^{\circ}$, respectively. The spot's temperature is always set to $3500 \mathrm{~K}$.

reconstruction in Fig. A.1 (appendix Test $5, S / N=500$ ), i.e. with molecules, is only marginally different. From this comparison, we conclude that molecules play no dominant role at the photospheric temperature of $5000 \mathrm{~K}$ and that our method is limited to a temperature recovery of approximately $\pm 100 \mathrm{~K}$.

The synthetic spectra in Fig. 1 show that most molecular contributions become only dramatically different for models cooler than about $4250 \mathrm{~K}$. Therefore, we performed a test with a much cooler star of $T_{\text {phot }}=4250 \mathrm{~K}$, corresponding to K7-M0 (Fig. 4). Only the more critical test case Star 1 was used. At that temperature, the many molecular lines depress the continuum level of the forward spectra by $10 \%$ (cf. top panels in Fig. 4). Note that this is a factor of 10 larger than what we assumed for the trial reconstructions in Test 23-26 in Fig. A.3 of the Appendix. A recovery without a molecular contribution is impossible with OPC and does not converge to a realistic solution. If molecules are included, OPC quickly converges and the surface map is surprisingly well recovered, as shown in Fig. 4. Just three rather weak, artificial spots with $\Delta T \approx 100 \mathrm{~K}$ appear at the lower rim of the visible stellar surface. The true spots are a. Atomic reconstruction of Star 1
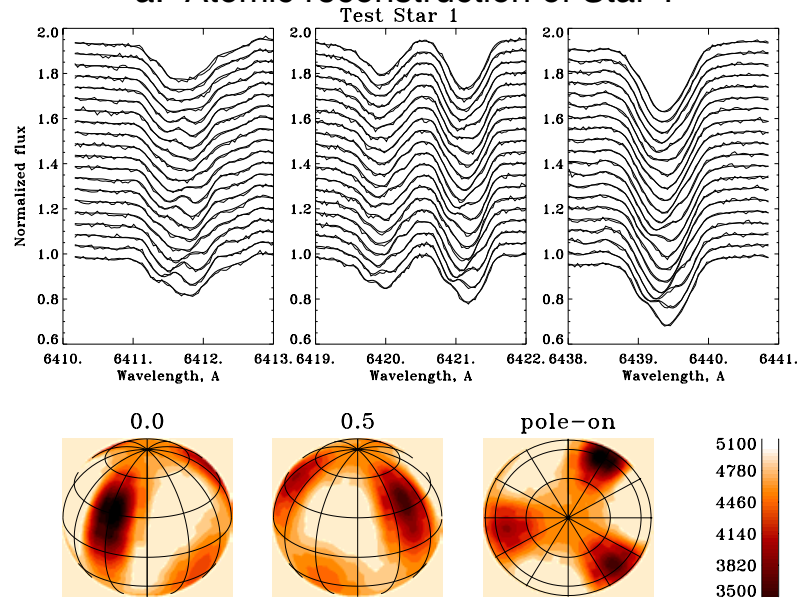

b. Atomic reconstruction of Star 2

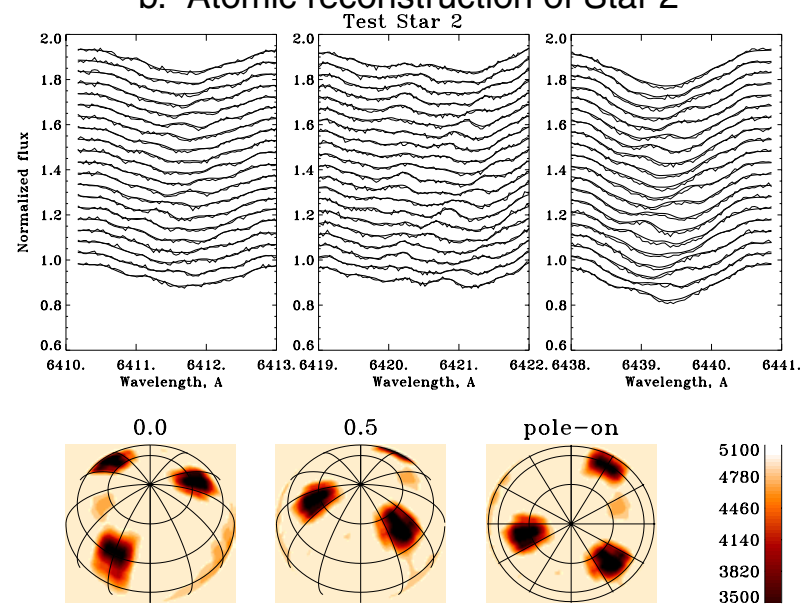

Fig. 3. Test reconstructions neglecting molecular lines. Test stars a) S1 and b) S2 were used with $\log g=4.5$ to mimic a true K2 mainsequence star. The forward computations were done with the full atomic and molecular line lists but the recovery is without molecular lines. All three wavelength regions were inverted simultaneously. The images for case $\mathrm{S} 1$ in a) show overally larger and less cooler spots compared to the input image, while the images for case S2 in b) recover nearly the correct temperature distribution with just a few lowcontrast artifacts.

recovered with an artificial penumbra-like structure and their average temperature is about $120 \mathrm{~K}$ warmer than the input model spot. The high-latitude spot is again the least well recovered and appears warmer by $\approx 300 \mathrm{~K}$ with respect to the model spot. Otherwise, the position and relative size of the features is well reconstructed.

More trial inversions of the K7/M0 test, but with effective temperatures of $4750 \mathrm{~K}$ and $4500 \mathrm{~K}$, also did not lead to convergence at the $97.5 \%$ probability although a formal solution was obtainable at $4750 \mathrm{~K}$ for both stellar cases (and also for $4500 \mathrm{~K}$ for case S2 but not S1). Any OPC inversion that completely neglects the existence of molecules at an effective temperature of $\approx 4250 \mathrm{~K}$ does not lead to a realistic solution. 


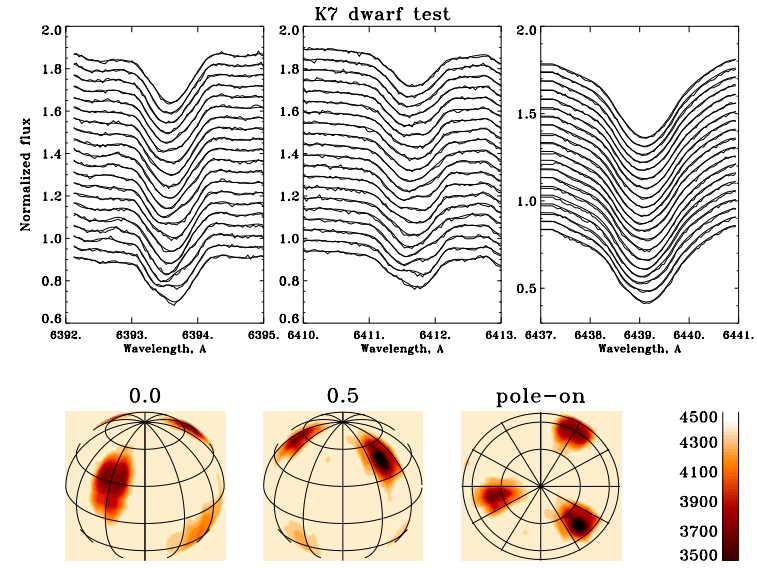

Fig. 4. The K7/M0-star test. Forward and inverse computations are done with molecular lines. The thin lines in the top panels show the artificial data of Star 1 but with $T_{\text {phot }}=4250 \mathrm{~K}, \log g=4.5, v \sin i=$ $24 \mathrm{~km} \mathrm{~s}^{-1}, i=60^{\circ}$ and $S / N$ of $200: 1$. The thick lines are the fits with our OPC inversion. The lower panels show the reconstructed images. Notice the strong continuum depression due to molecules in the top panels, e.g., the 6393-Å region appears with a depression of $10 \%$.

Table 2. Summary of systematic parameter tests. The running number, \#, corresponds to the test number in Figs. 5-7.

\begin{tabular}{|c|c|c|c|c|}
\hline$\#$ & $T_{\mathrm{ph}}, \mathrm{K}$ & $V \sin i$ & $S / N$ & spectral region \\
\hline \multirow[t]{2}{*}{1} & 5000. & 50. & 200 & $6410-6445 \AA$ \\
\hline & & & & FeI and $\mathrm{CaI}$ lines \\
\hline \multirow[t]{2}{*}{2} & 5000. & 24. & 200 & $6410-6445 \AA$ \\
\hline & & & & $\mathrm{FeI}$ and $\mathrm{CaI}$ lines \\
\hline \multirow[t]{2}{*}{3} & 5000 & 36. & 200 & $6439 \AA 7051-7059 \AA$ \\
\hline & & & & $\mathrm{CaI}, \mathrm{TiO}$ and $\mathrm{CN}$ lines \\
\hline \multirow[t]{2}{*}{4} & 5000 & 36. & 200 & 7049-7059 ̊ \\
\hline & & & & $\mathrm{CaI}, \mathrm{TiO}$ and $\mathrm{CN}$ lines \\
\hline \multirow[t]{2}{*}{5} & 5000. & 24. & 200 & $6160-6200 \AA$ \\
\hline & & & & FeI, NiI and VI lines \\
\hline \multirow[t]{2}{*}{6} & 5000. & 24. & 50 & $6160-6200 \AA$ \\
\hline & & & & FeI, NiI and VI lines \\
\hline \multirow[t]{2}{*}{7} & 4250. & 24. & 300 & $15567-15574 \AA$ \\
\hline & & & & $\mathrm{OH}$ band \\
\hline \multirow[t]{2}{*}{8} & 4250. & 24. & 300 & 23 404-23 411 Å \\
\hline & & & & $\mathrm{CO}$ band \\
\hline
\end{tabular}

\subsection{Systematic parameter tests}

The input parameters of more systematic tests are summarized in Table 2. For the first six tests, the default is Star 1 in Table 1 but with $\log g=3.5$ and with values of $v \sin i$ of 24, 36 and $50 \mathrm{~km} \mathrm{~s}^{-1}$. The cooler Star 3 model $\left(T_{\text {phot }}=4250 \mathrm{~K}\right)$ with $\log g=4.5$ was used in Tests 7 and 8 . The resulting images and fits to the simulated spectra are shown in Figs. 5 and 6.

For the first two runs, synthetic spectra were calculated in the wavelength region $6410-6445 \AA$ containing Fe I and $\mathrm{Ca} I$ lines which are often used for the surface imaging of cool stars. For the reconstruction, we worked with "data" of signal-to-noise ratio of 200:1 and from twenty equally spaced phases. The result of this basic reconstruction is presented in Fig. 5 (Test 1) for $v \sin i=50 \mathrm{~km} \mathrm{~s}^{-1}$, and in Fig. 5 (Test 2) for $v \sin i=24 \mathrm{~km} \mathrm{~s}^{-1}$. The positions of the spots, their projected area and their contrast are well determined for the assumed spatial resolution but Test 1 recovered more artifacts at $\Delta T \approx 200 \mathrm{~K}$ than Test 2 . This is solely due to the different (effective) velocity grid size for the two tests.

For the next test, Test 3, we calculate the synthetic spectra for two spectral regions containing the Ca I $6439 \AA$ line and the $\mathrm{TiO} \gamma(0,0) R_{3}$ band head at 7051-7059 $\AA$. The latter region also contains atomic lines and $\mathrm{CN}$ lines which can be important contributors at temperatures of 4500-5000 K. The image restoration is done simultaneously for both wavelength regions. In our calculations all available data for $\mathrm{CH}$ and for the red system of $\mathrm{CN}$ (SCAN database line lists) and for the violet system of $\mathrm{CN}$ and for $\mathrm{OH}, \mathrm{NH}, \mathrm{SiO}, \mathrm{SiH}, \mathrm{MgH}$, $\mathrm{C}_{2}$ and $\mathrm{CO}$ (Kurucz CD) are used. For $\mathrm{TiO}$ we used data from Plez (1998), which can be found at ftp.astro.uu.se. Comparing the maps in Fig. 5, we find in general good agreement with the input image except that the spot at longitude $115^{\circ}$ was reconstructed with a temperature difference of $1200 \mathrm{~K}$ instead of $1500 \mathrm{~K}$. Note that more quantitative comparisons are given for a large number of detailed tests in the Appendix in Table A.1.

For the reconstruction in Test 4, we adopt solely the spectral region $7049-7059 \AA$, dominated by the $\mathrm{TiO} \gamma(0,0) R_{3}$ band head. Again, the agreement between simulated and restored spectra is very good but more artifacts at the $\Delta T \approx 200 \mathrm{~K}$ level appear.

According to the investigations by Berdyugina et al. (1998), Saar \& Osten (1996) and Strassmeier \& Rice (1998) the $6160 \AA$ spectral region with various temperature sensitive lines of $\mathrm{Fe} I$, $\mathrm{Ni} I$ and V I can be effectively used for the image restoration. Tests 5 and 6 were performed for several lines from this region, taking into account many blends from atomic and molecular lines. The reconstruction in Test 5 appears to be very similar to the one in Test 2 from the 6410-6445 region, its overall error budget does not exceed $\approx 300-400 \mathrm{~K}$ (see also Fig. 7). From Test 6 one can conclude that the spots and the spot locations are well determined even in the case of $S / N$ as low as 50:1, despite the many artifacts.

Two further tests (Test 7 and 8) were performed for the infrared $\mathrm{OH}$ and $\mathrm{CO}$ lines which, like the TiO lines, can improve the diagnostic capability of atomic lines. The input image has now a photospheric temperature of $4250 \mathrm{~K}$, i.e. a K7/M0 spectral type. Additionally, the three-spot model was replaced with the two-spot spot model of Star 3 with areas larger by a factor of two in order to produce more significant variations in the broadened line profiles. The input map is shown in Fig. 6. We simulated data in the $15567-15574 \AA$ region with the $\mathrm{OH}$ first-overtone vibration-rotation band. Molecular data are from Goldman et al. (1998) with the dissociation energy being $D_{0}=4.392 \mathrm{eV}$ (Huber \& Herzberg 1979). Second, we calculated spectra for the 23 404-23411 ̊ region which includes many lines of the $\mathrm{CO}$ first-overtone vibrationrotation bands. The wavelengths, excitation potentials and $g f$-values for CO were published by Goorvitch (1994). 

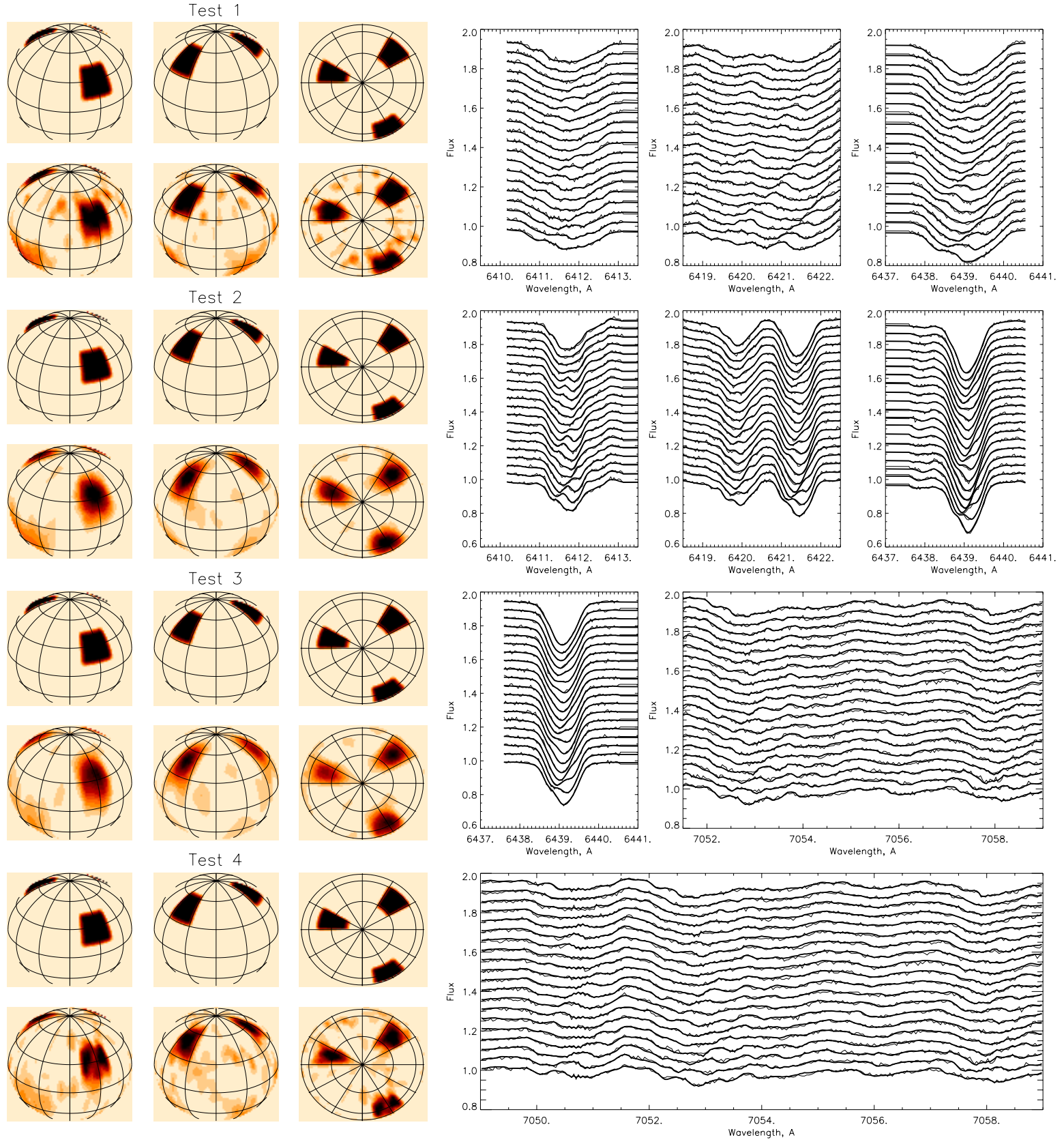

Fig. 5. Restored images of Test 1-4. The parameters of each test are given in Table 2. Maps are shown in the left panels. Its top row is the artificial input map, the bottom row its reconstruction. Simulated "observed" data are shown in the right panels by thin lines, while thick lines are the calculated line profiles based on the reconstructed image. Individual spectra are shifted in the vertical direction; phase is increasing from top to bottom; wavelengths are indicated. Test 1 and 2 are mainly based on three atomic lines. Test 3 on one atomic and the TiO $\gamma(0,0) R_{3}$ band head, and Test 4 only on the TiO band head. The color/grey scale of Fig. 2 (top) applies to all images.

The CO dissociation energy was taken as $11.090 \mathrm{eV}$. Both wavelength regions produced a satisfactory reconstruction of the spots in the input image but with the addition of an artificial equatorial band of $\Delta T \approx 150 \mathrm{~K}$.

\section{Results and discussion}

Assuming that the stellar parameters are known precisely, the number of free parameters has to be of the order of the number of stellar surface grid points. In most of our numerical verifications, an inversion grid of $6^{\circ} \times 6^{\circ}$ on the stellar surface with $n=1500$ pixels was used. In the general case the solution is constrained by the number $m$ of input data points and this can lead to an under-determined situation. On the contrary, for the approach developed by Terebizh (2004) as well as in the Occamian approach (Terebizh 1995; Berdyugina 1998), only a part of the principal components $p_{\mathrm{j}}, j=1 \ldots r$, with $r<n$, completely describes the object $x_{0}$ (our Eqs. (1) and (3)). Principal components $p_{\mathrm{j}}$ with $r<j<n$, contain no significant information but only noise. These can be excluded from the transformation in Eq. (3) by zero padding (Press et al. 1992; Berdyugina 1998) or by applying corresponding 

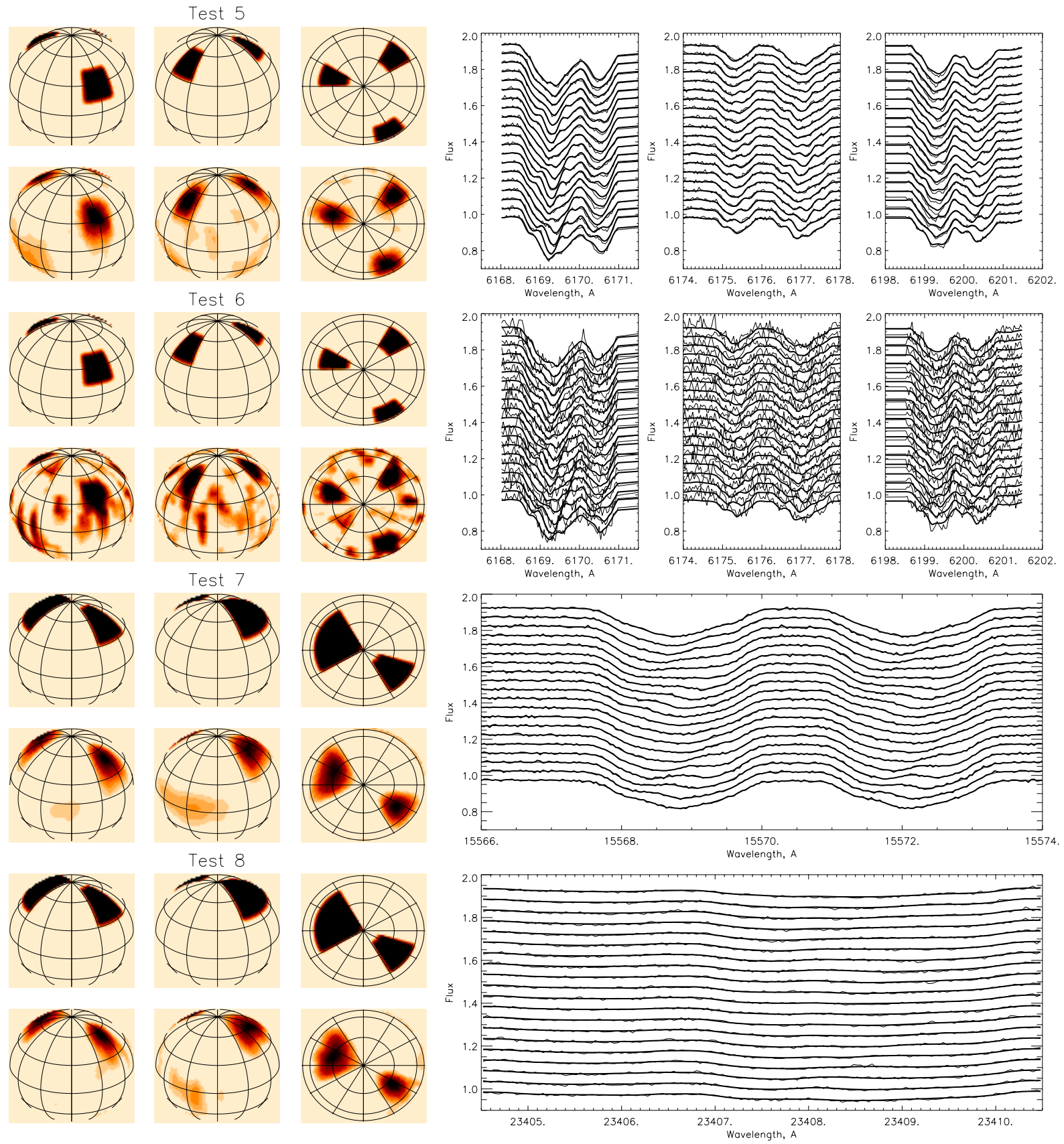

Fig. 6. Restored images of Test 5-8. As in Fig. 5, the test numbers refer to the running number in Table 2 . Tests 5 and 6 are mainly based on three atomic-line regions (Test 6 with $S / N=50: 1$ ), Test 7 on the $\mathrm{OH}$ first-overtone vibration-rotation band, and Test 8 on the appropriate CO-band lines. Note that for the images in Test 7 and 8, the color/grey scale of Star 3 in Fig. 2 applies.

filtering (Eq. (4)). Depending upon the model case the number of "effective" $p_{\mathrm{j}}$ in our tests is in the range of 600-900, while the total number points in the data vector $y_{0}$ is usually several times larger than this.

Reduction of the signal to noise ratio from 200:1 to 50:1 leads to the result that the restored image becomes significantly blurred and the contrast reduced, but the position of the spots can be determined very well. Rice \& Strassmeier (2000) noted that the case of $S / N$ less than 75:1 has never been used for real applications as long as least-squares deconvolution was implemented to boost $S / N$ ratios from 45 to 1000 (e.g. Barnes et al. 1998). We suppose that for low values of $S / N$, surface imaging based on co-added line profiles can be supplemented by our restoration using the principal component analysis from even broader spectral regions including many spectral features simultaneously.

Our consideration of the Fisher information matrix gives us the possibility to estimate the lower limit of the variance matrix of the solution $x$. Terebizh (1995) showed that in this case the variances of the solution can be found as

$\sigma^{2}(x) \geqq \operatorname{Diag}\left[I^{-1}\right]$.

We calculated the inverted information matrix for all our numerical verification tests but present graphics of the error 

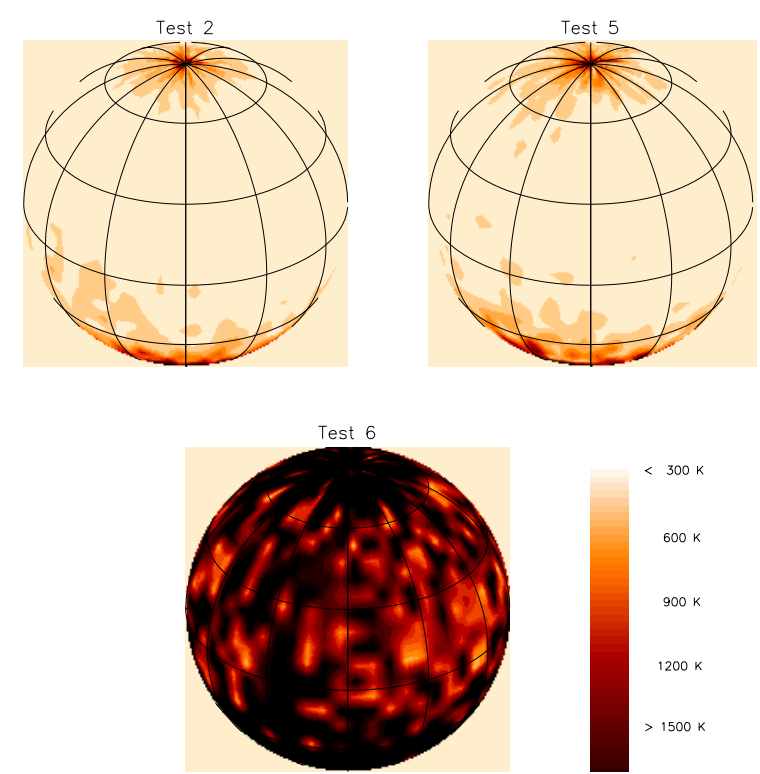

Fig. 7. Error distribution on the stellar surface for the restoration of the test star image of Tests 2, 5 and 6 in Figs. 5 and 6.

distributions only for Tests 2, 5 and 6 in Fig. 7. For all cases, the error distribution $\sigma(x)$ is found to be a function of latitude and is dominated by the pole-to-equator distribution. It has minimum values in the region close to the sub-observer latitude on the stellar surface. For example, in Test 1 the area with the estimation of $\sigma(x) \geqq 150-300 \mathrm{~K}$ extends to the latitudes between $0^{\circ}$ and $+60^{\circ}$, while for the polar region and latitudes less than $-30^{\circ}$ the errors are quite large (from $350-400 \mathrm{~K}$ up to $1500 \mathrm{~K})$. Similar behavior of the error distribution was also found by Piskunov \& Kochukhov (2002) who obtained it from the main diagonal of the inverse of the curvature matrix while, according to the results obtained by Berdyugina (1998), their minima are located on the visible rotational pole. We confirmed the results for the error distribution in Test 2 with the corresponding analysis for Test 5 , which differs only in the spectral region used for the restoration. Reduction of the $S / N$ ratio from 200:1 to 50:1 strongly affects the level of variance, as seen in Test 6 . Our formal error estimates are determined mostly by the amount of information contained in the set of line profiles as well as by the degree of linear dependency of the parameters. If, e.g., $T_{\text {phot }}$ is only known with some uncertainty, the real errors would be larger.

\section{Conclusions}

Several characteristics of the new OPC approach by Terebizh (2004) significantly influence our Doppler imaging procedure. In detail, we conclude the following.

1. The method of quasi-optimal filtering of principal components produces stable and efficient solutions by relying only on the internal resources of the inverse theory, namely on the assumption about the structure of the optimal estimate.

2. Non-monotonic behavior of the quasi-optimal filter is different from the one for the truncated estimate (Press et al. 1992; Berdyugina 1998). The quasi-optimal filter leaves in the object's estimate only those principal components that have the highest accuracy of restoration.

3. Use of the refined image $\phi$ of length $n$ instead of an initial dataset $y_{0}$ of size $m$, with $m>n$, allows us to reduce the amount of computations.

4. Simulations in which the stellar parameters are supposed to be known precisely indicate that our "formal" error estimates are determined mostly by the amount of information contained in the set of line profiles and by the degree of linear dependency of the parameter(s). The errors quoted are not errors in the usual sense but describe the inherent information content of the reconstructed image.

5. Molecular input is important for stars with cool spots with temperatures below $4000 \mathrm{~K}$ and becomes mandatory for stars with effective temperatures of and below $4250 \mathrm{~K}$. Even for the unspotted photosphere of $5000 \mathrm{~K}$ precise line profile calculations should take into account the absorption from $\mathrm{CN}, \mathrm{CH}$, etc.

6. The inverse problem is nonlinear for many parameters. Ideally, the simultaneously inverted spectral lines should show the same sensitivity to such nonlinearities, then we would get the most stable solution.

7. Calculations of opacities and intensities of atomic and molecular lines are included in our code but must rely on the correctness of its values.

8. We have not dealt with the uncertainties on the real values of atomic and molecular input parameters. Analyzing real observations, such uncertainties may produce significant errors in the restored images. Our strategy of using a wide range of spectral regions with thousands of blends does not allow us to discuss the influence of errors of transition probabilities or damping constants for a certain molecular or atomic line, or the main contributor to a particular blend, as was done previously in works by Rice \& Strassmeier (2000). Therefore, the current line lists are to be verified against high-quality spectra of standard stars with well established atmospheric parameters and normal chemical composition.

Acknowledgements. We are very grateful to Dr. V. Y. Terebizh for the numerous scientific discussions, consultations and useful practical advices. This work was supported by the German Deutsche Forschungsgemeinschaft, DFG project number STR645/1-1.

\section{References}

Barnes, J. R., Collier Cameron, A., Unruh, Y. C., Donati, J.-F., \& Hussain, G. A. I. 1998, MNRAS, 299, 904

Berdyugina, S. 1998, A\&A, 338, 97

Berdyugina, S. 2002, in 12th Workshop, Cool Stars, Stellar Systems, and the Sun, ed. A. Brown, G. Harper, \& T. Ayres, www-publication, 210

Berdyugina, S. V., Berdyugin, A. V., Ilyin, I., \& Tuominen, I. 1998, A\&A, 340, 437

Collier Cameron, A. 1992, in Surface inhomogeneities on late-type stars, ed. P. Byrne, \& D. Mullan (Berlin: Springer Verlag), 33

Collier Cameron, A. 2001, in Lecture Notes in Physics (Springer Verlag), 573, 183

Donati, J.-F., \& Brown, S. F. 1997, A\&A, 326, 1135

Goldman, A., et al. 1998, JQSRT, 59, 453 
Goorvitch, D. 1994, ApJS, 95, 535

Gray, D. F. 1992, The observation and analysis of stellar photospheres (Cambridge: CUP)

Gray, R. O. 2000, http://www.phys.appstate.edu/

Hackman, T., Jetsu, L., \& Tuominen, I. 2001, A\&A, 374, 171

Hotelling, H. 1933, J.Educ.Psych., 24, 417

Huber, K.-P., \& Herzberg, G. 1979, Constants of Diatomic Molecules, Van Norstand, New York

Kupka, F., Piskunov, N., Ryabchikova, T. A., Stempels, H. C., \& Weiss, W. W. 1999, A\&AS, 138, 119

Kurucz, R. L. 2000, http: //www. cfaku5 .harvard.edu

Kurucz, R. L., \& Bell, B. 1995, Atomic Line List, CD-ROM No. 23, Smithonian Astroph. Obs. Cambridge, MA

O’Neal, D., Neff, J. E., \& Saar, S. H. 1998, ApJ, 507, 919

O'Neal, D., Neff, J. E., Saar, S. H., \& Cuntz, M. 2005, AJ, 128, 1802

Piskunov, N. E., \& Kochukhov, O. 2002, A\&A, 381, 736

Piskunov, N. E., Kupka, F., Ryabchikova, T. A., Weiss, W. W., \& Jeffery, C. S. 1995, A\&AS, 112, 525

Piskunov, N. E., \& Rice, J. B. 1993, PASP, 105, 1415

Plez, B. 1998, A\&A, 337, 495

Press, W. H., Teukolsky, S. A., Vetterling, W. T., \& Flannery, B. P. 1992, Numerical Recipes (Cambridge: Cambridge Univ. Press)

Rice, J. B. 2002, AN, 323, 220
Rice, J. B., \& Strassmeier, K. G. 2000, A\&AS, 147, 151

Saar, S. H., \& Osten, R. A. 1996, MNRAS, 284, 803

Strassmeier, K. G. 2002, AN, 323, 309

Strassmeier, K. G., Kratzwald, L., \& Weber, M. 2003, A\&A, 408, 1103

Strassmeier, K. G., Lupinek, S., Dempsey, R. C., \& Rice, J. B. 1999, A\&A, 347, 212

Strassmeier, K. G., \& Rice, J. B. 1998, A\&A, 330, 685

Savanov, I. S. 2002, in Sunspots and Starspots, Poster proceedings 1st Potsdam Thinkshop, ed. A. Washüttl, \& K. G. Strassmeier (AIP), 3

Savanov, I. S. 2005, in High Resolution Infrared Spectroscopy, ed. H. U. Kaufl, R. Siebenmorgen \& A. Moorwood, ESO Workshop, 431

Terebizh, V. Y. 1995, Physics - Uspekhi 38, 137

Terebizh, V. Y. 2004, A\&AT, 23, 85

Terebizh, V. Y., \& Biryukov, V. V. 1994, Ap\&SS, 218, 65

Tsymbal, V. 1996, in Model Atmospheres and Spectrum Synthesis, ed. S. J. Adelman, F. Kupka, \& W. W. Weiss, ASPC, 108, 198

Valenti, J., Piskunov, N., \& Johns-Krull, C. 1998, ApJ, 498, 851

Vogt, S. S., Penrod, G. D., \& Hatzes, A. P. 1987, ApJ, 321, 496

Wallace, L., Livingston, W., Bernath, P. F., \& Ram, R. S. 1999, An atlas of the Sunspot umbral spectrum, NSO/NOAO 


\section{Online Material}




\section{Appendix A: Detailed tests and discussion}

The following sections and figures present and discuss further numerical tests. The total number of tests was 46. Graphical results are summarized in Figs. A.1-A.4. The left column in Figs. A.2-A.4 is always for Star 1, the right column for Star 2, according to Table 1. Figure A.1 additionally shows the error estimates as an average value in the longitudinal direction. These error graphs are omitted in the other figures but Table A.1 lists the average values for three representative latitudes. All OPC results are in good agreement with those discussed by Piskunov \& Rice (1993), Berdyugina (1998) and Rice \& Strassmeier (2000) and complement their results.

\section{A.1. Test 1-6: signal-to-noise ratio}

Figure A.1 shows the first three tests from spectra with different signal-to-noise ratios ( $S / N$ equal to 200:1, 500:1 and 60:1). The high inclination case (Star 1) was found to be more stable to the $S / N$ changes than the low inclination case (Star 2). However, the level of average errors for the regions close to the sub-observer point are only slightly better for Star 1 than for Star 2 . We find deviations of $60-100 \mathrm{~K}$ for the $S / N=200$ cases (for S1 and S2) and 10-60 K (S1) and 20-80 K (S2) for the $S / N=500$ case. Average errors are shown in the right panels of Fig. A.1. Because the $\sigma(x)$ deviations contain mostly latitude dependency (examples of deviation maps can be found in Fig. 7) we averaged them in the longitudinal direction and estimated the scatter within latitude belts by computing standard deviations for each belt (as did, e.g., Piskunov \& Kochukhov 2002).

The restored image for the case $\mathrm{S} 2$ for $S / N=200$ becomes slightly more blurred than in the $\mathrm{S} 1$ case. We emphasize this test, as the case of $S / N$ ratio of 200:1 represents the most typical situation of current observational data. $S / N$ ratios equal to 500:1 and above are mostly left to future observations of bright stars with large telescopes, e.g., with PEPSI at the LBT. Restoration for the low $S / N$ ratio of $60: 1$, so far never used in practice except in combination with least-squares deconvolution techniques, is possible and also turned out to be better for the high-inclination case than for the low-inclination case. For the S2 test, the restored image is more blurred, although in both cases the position and the contrast of the spots is well determined. The absolute error level is quite large, $\Delta T$ of order 500-900 K. Its latitudinal dependency is slightly different for S1 and S2 though. We can conclude that, even for the case of low values of $S / N$ of the order of 50-70, our restoration procedure using principal component analysis can be used for the determination of the geometrical position of the spots.

Because the $\sigma(x)$ error deviations have minimum values in the regions close to the sub-observer latitude, we do not present additional plots of the $\sigma(x)$ with latitude but characterize their distribution by just three typical values: one that represents the sub-observer latitude and the other two are for belts with latitudes centered at $+81^{\circ}$ and $-51^{\circ}\left(-21^{\circ}\right.$ for the low-inclination $\mathrm{S} 2$ case). These estimates, together with their standard deviations, are listed in Table A.1.

\section{A.2. Test 7-14: rotational velocity and inclination}

Figure A.2 compares reconstructions for S1 and S2 when either the projected rotational velocity, $v \sin i$, or the inclination of the rotational axis, $i$, was altered with respect to the input data (see also Rice 2002). Changes of the rotational velocity of $\pm 2 \mathrm{~km} \mathrm{~s}^{-1}$, i.e. $8 \%$ of the nominal value in Table A.1 for case $\mathrm{S} 1$ and $4 \%$ for case S2, led to the appearance of a polar cap or an equatorial belt. A too low a $v \sin i$ for $\mathrm{S} 1$ causes a polar cap cooler by $400 \mathrm{~K}$ than the photospheric temperature while too high a value causes a polar cap warmer by $350 \mathrm{~K}$. As expected, the same is true for the low-inclination case S2 but that the comparable temperature differences are smaller; 100-200 K when $v \sin i$ is too low, and $250 \mathrm{~K}$ when $v \sin i$ is too high. Spot locations are always well determined, especially in case $\mathrm{S} 2$.

Changes of the inclination angle by $\pm 12^{\circ}$ (for $\mathrm{S} 1$ ) and $\pm 6^{\circ}$ (for S2) also led to well defined spots but, in the $\mathrm{S} 1$ case for $i_{\mathrm{S} 1}=i_{\text {nominal }}-12^{\circ}=48^{\circ}$, the spot contrast appeared reduced by $400-500 \mathrm{~K}$. On the other hand, the images for the lowinclination case (S2) suffer from a number of spurious smallscale spots while the spot contrast was correctly reconstructed. This is especially true for the $i_{\mathrm{S} 2}=i_{\text {nominal }}+6^{\circ}=36^{\circ}$ image in Fig. A.2.

\section{A.3. Test 15-18: photospheric temperature}

Our tests indicate that an error of $\pm 100 \mathrm{~K}$ in $T_{\text {phot }}$ can significantly affect the solution for case $\mathrm{S} 1$, where the position of spots in latitudinal direction becomes poorly determined and smeared towards the stellar equator. This was also noticed by Strassmeier et al. (2003) for the tests with TEMPMAP for HD 31993 and was attributed to the non-linear dependency on various other spectrum parameters that are kept constant during the inversion. For the case S2, the contrast of spots appears reduced but its latitudinal extent is more-or-less correctly reconstructed. We can also see that an underestimation of $T_{\text {phot }}$ by $100 \mathrm{~K}$ leads to a weak polar cap with $\Delta T \approx 50-60 \mathrm{~K}$, while overestimation leads to a warm cap with $\Delta T \approx 200 \mathrm{~K}$, possibly with a very weak cool equatorial belt. All these artifacts, except perhaps the latitudinal smearing, would probably go undetected in real data.

\section{A.4. Test 19-22: phase coverage}

Our nominal restorations were performed with optimal phase coverage, i.e. 20 data sets equidistantly distributed over the rotational period. We remove six successive phases (from 0.7 to 0.95$)$. This corresponds to a phase gap of 0.3 periods or $\approx 110^{\circ}$ in observations, clearly an exaggerated situation. For the high-inclination S1 case it obviously leads to a lowercontrast reconstruction of the spot that falls into the phase gap (Fig. A.3). For the low-inclination S2 case, a better restoration of the spots was achieved but a number of weak artificial spots appeared.

A worst case scenario was assumed for the next test when we consider only 6 randomly distributed observations over the entire period, i.e. spectra at phases $0.0,0.26,0.39,0.63,0.77$ and 0.86. Clearly, this test demonstrates that restoration for 
I. S. Savanov and K. G. Strassmeier: Surface imaging using atomic and molecular features. I., Online Material p 3

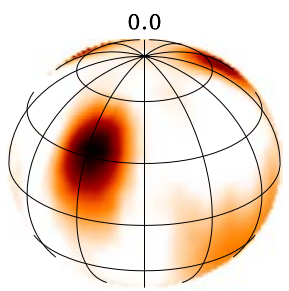

TEST $1 \mathrm{~S} / \mathrm{N}=200$
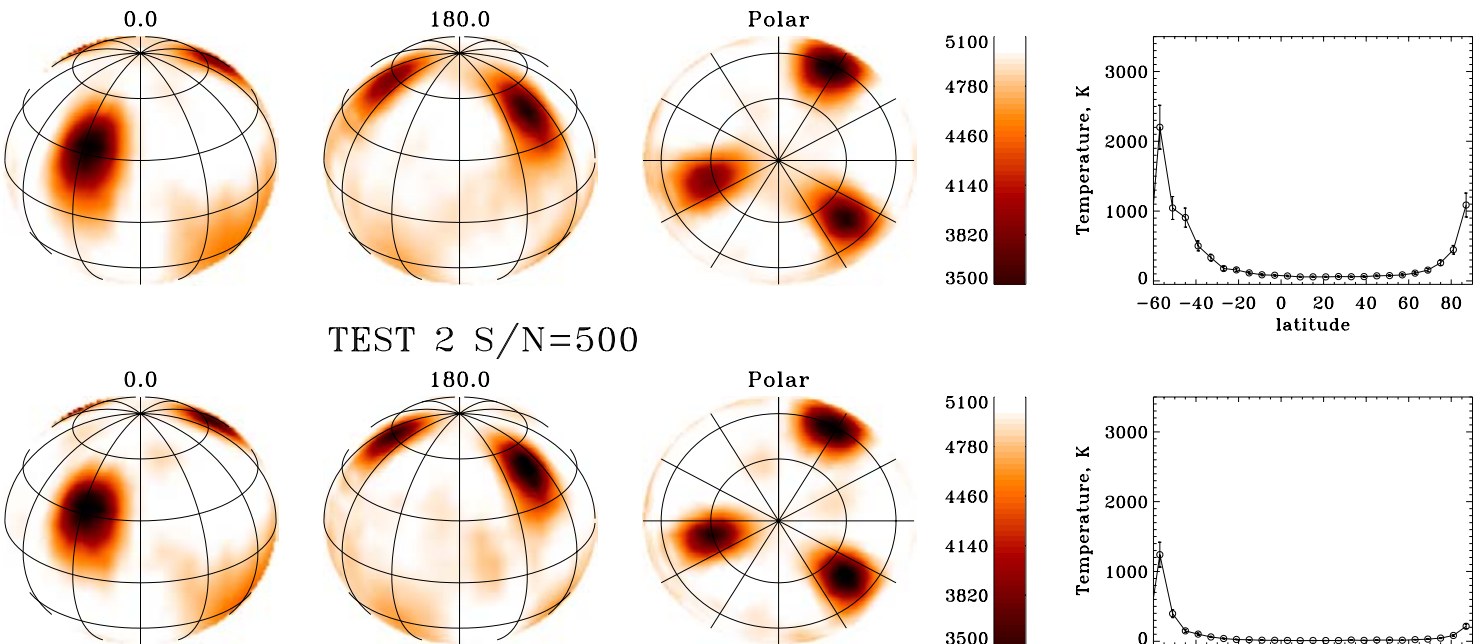

TEST $2 \mathrm{~S} / \mathrm{N}=500$
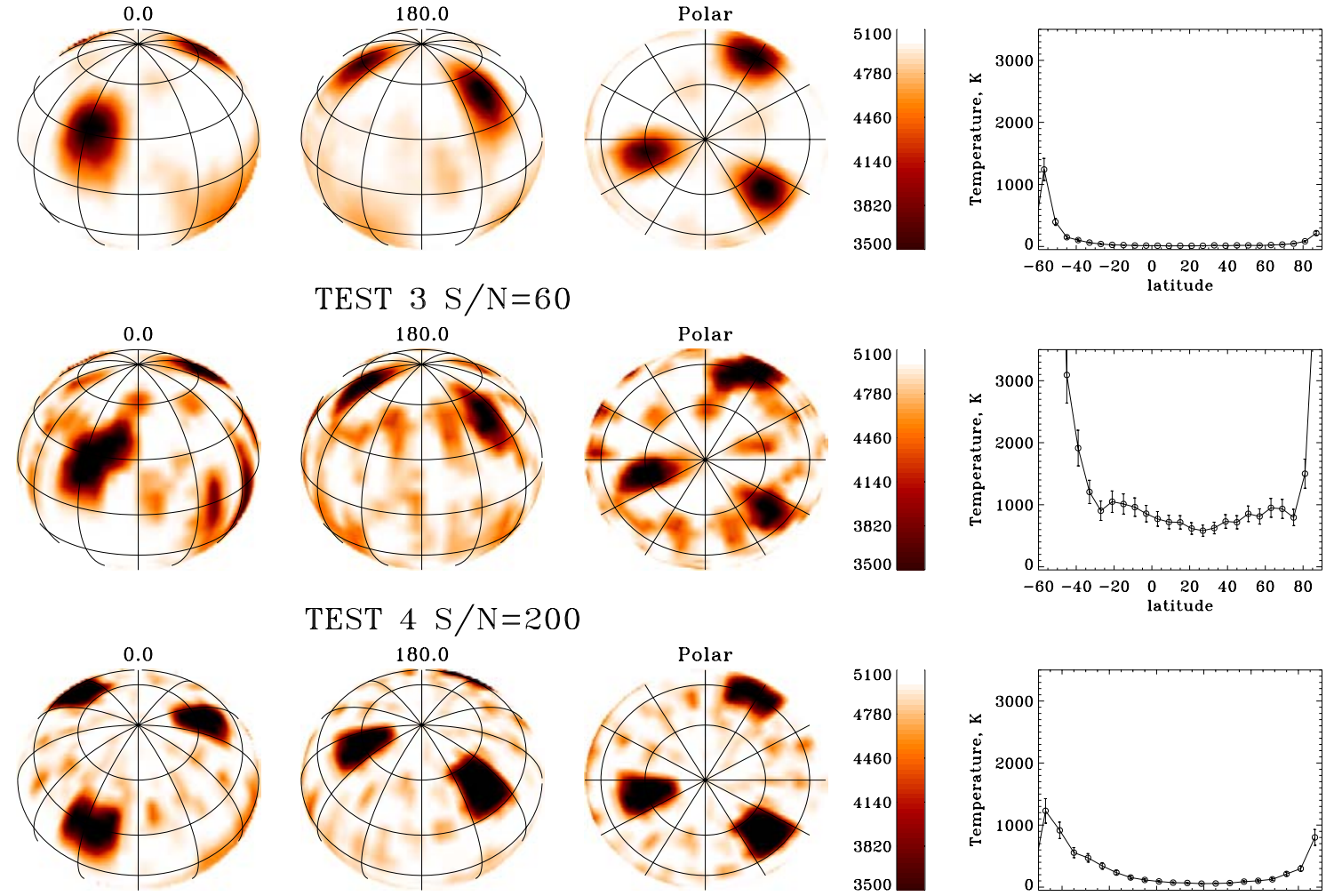

TEST $4 \mathrm{~S} / \mathrm{N}=200$
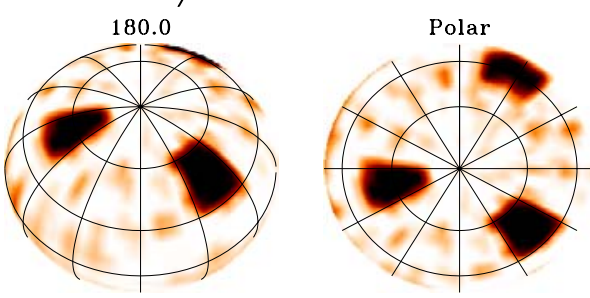

$\begin{aligned} & 5100 \\ & 4780 \\ & 4460 \\ & 4140 \\ & 3820 \\ & 3500\end{aligned} \mid$
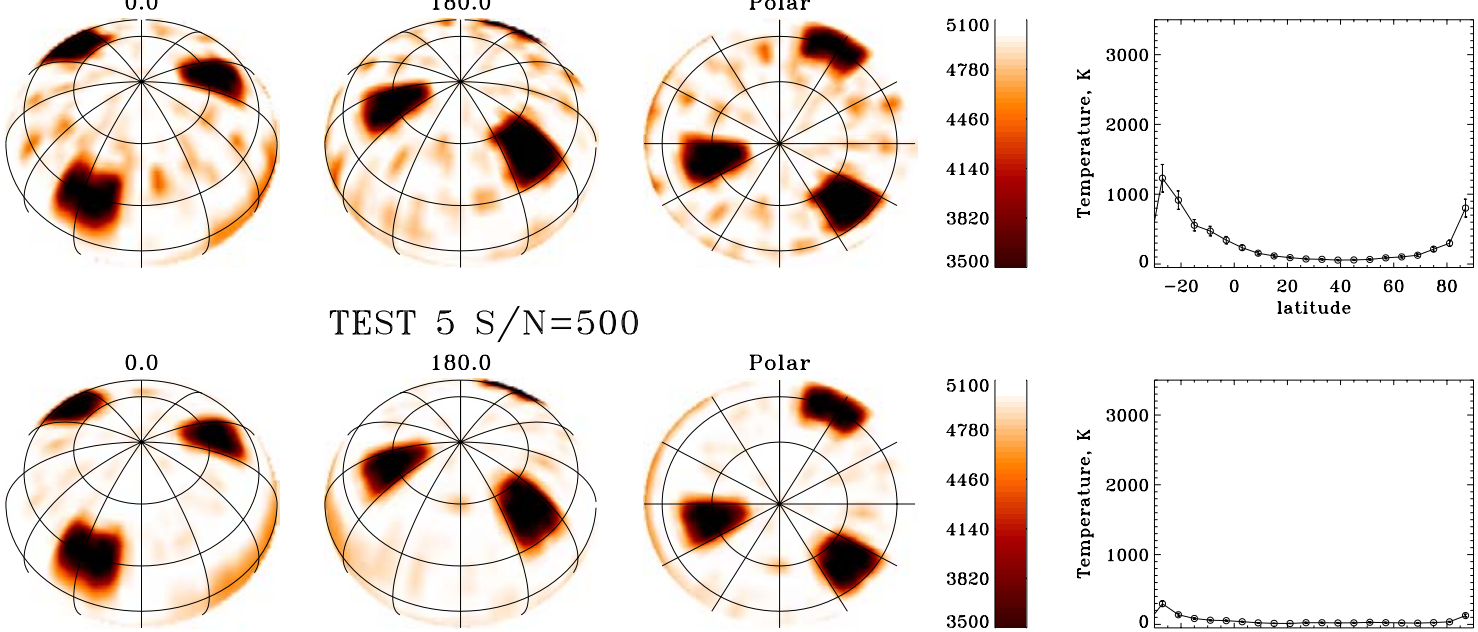

TEST $5 \mathrm{~S} / \mathrm{N}=500$
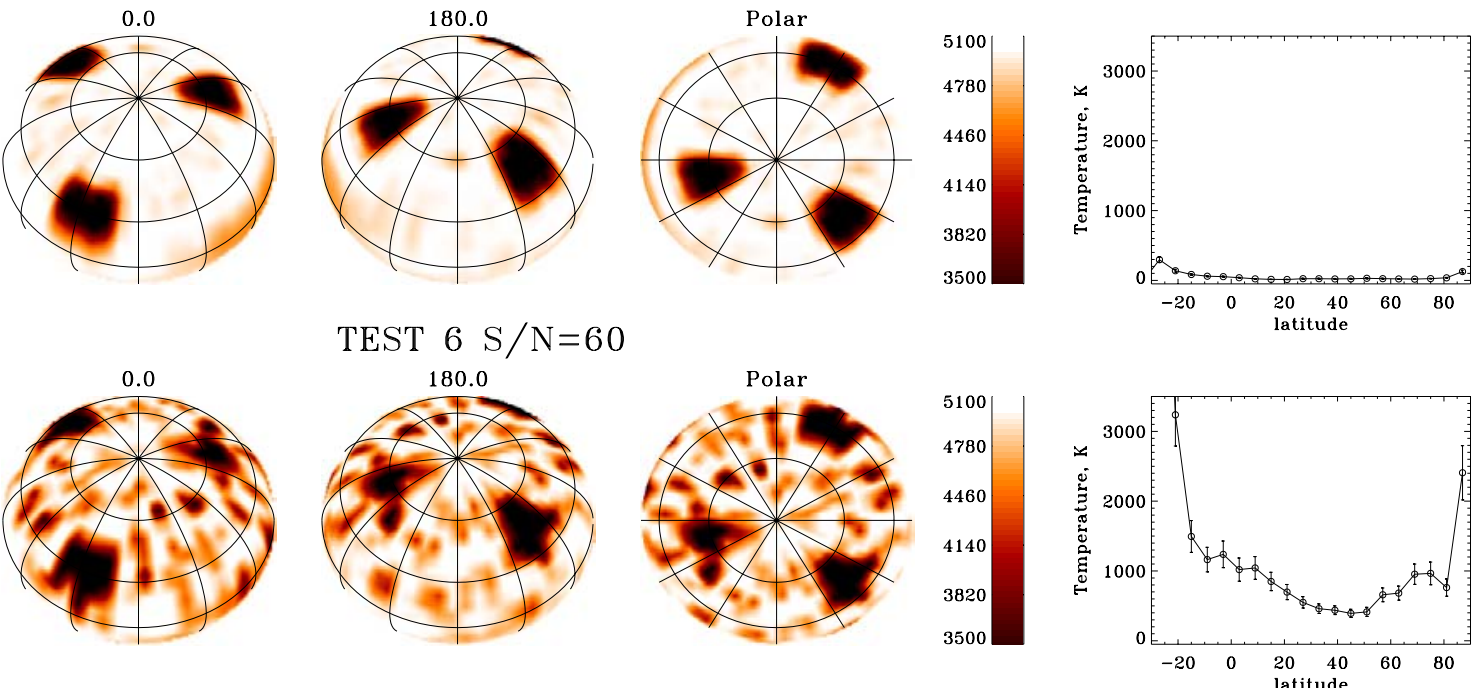

Fig. A.1. Test 1-6. Test reconstructions with different signal to noise $(S / N)$ ratio of the artificial data. The restored images are shown as spherical projections at longitudes of $0^{\circ}$ and $180^{\circ}$ (left two images in each row) and as a polar view (marked "polar"). The bars give the color code related to surface temperature. The right panels are the longitudinally averaged errors at all latitudes. Test star 1 is shown in Test 1-3, test star 2 for Test 4-6. 


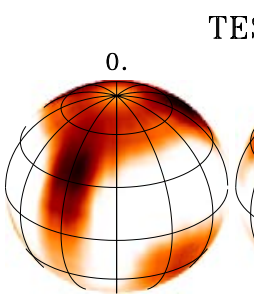

TEST $7 \mathrm{Vsini}=22 \mathrm{~km} / \mathrm{s}$
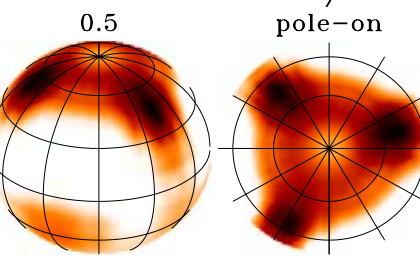

TEST 9 Vsini $=26 \mathrm{~km} / \mathrm{s}$
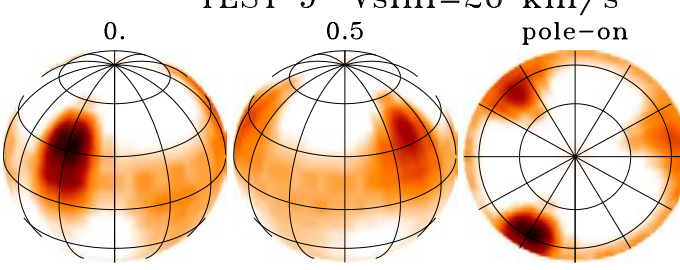

TEST $11 \mathrm{i}=48 \mathrm{deg}$
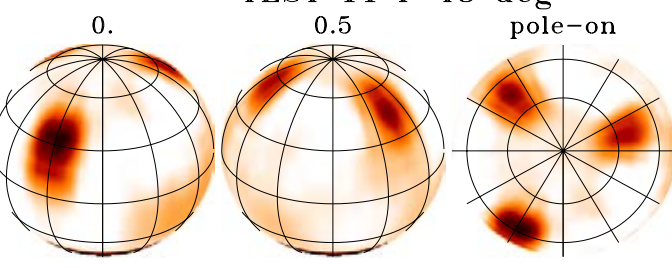

TEST $13 \mathrm{i}=72 \mathrm{deg}$
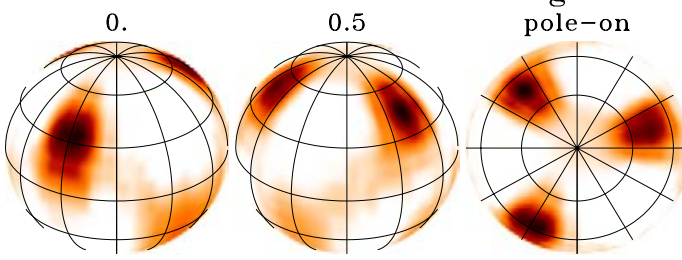

TEST $15 \mathrm{~T}=4850 \mathrm{~K}$
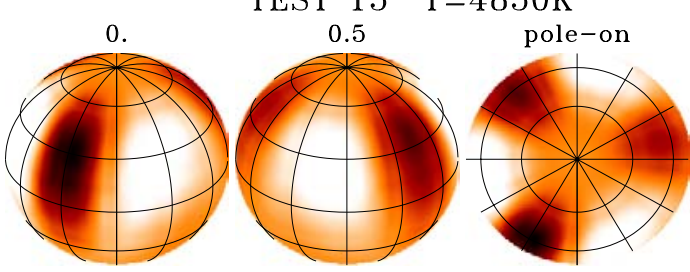

TEST $17 \quad \mathrm{~T}=5150 \mathrm{~K}$
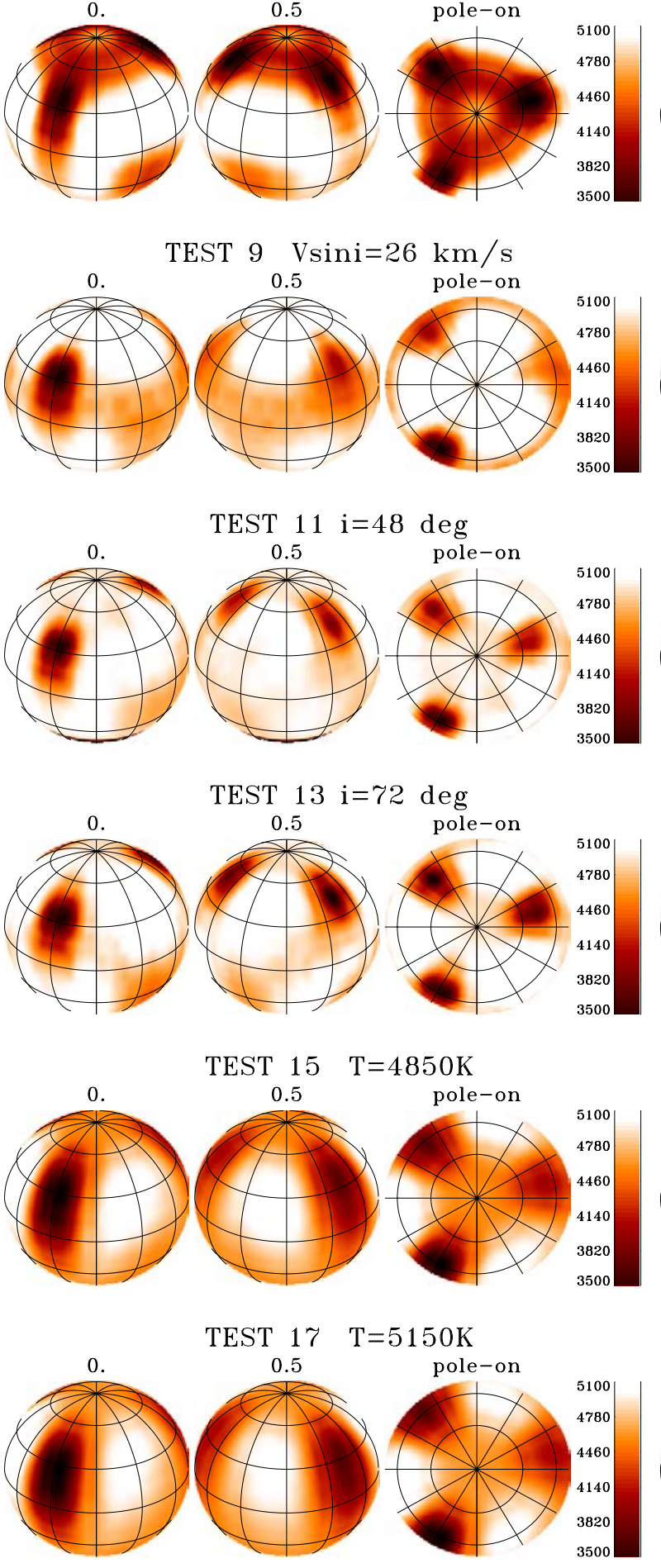

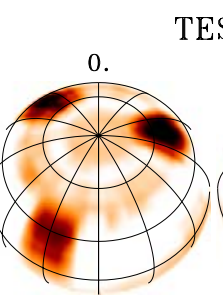

TEST $8 \mathrm{Vsini}=48 \mathrm{~km} / \mathrm{s}$
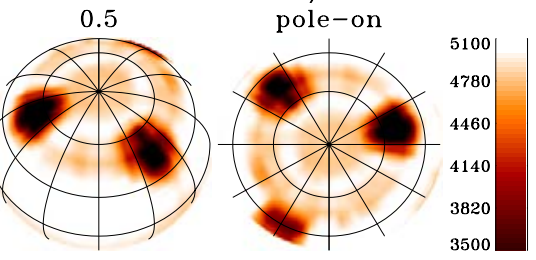

TEST $10 \quad \mathrm{~V} \sin \mathrm{i}=52 \mathrm{~km} / \mathrm{s}$
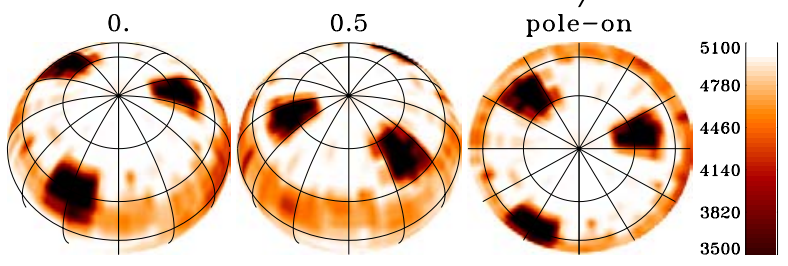

TEST $12 \mathrm{i}=24 \mathrm{deg}$
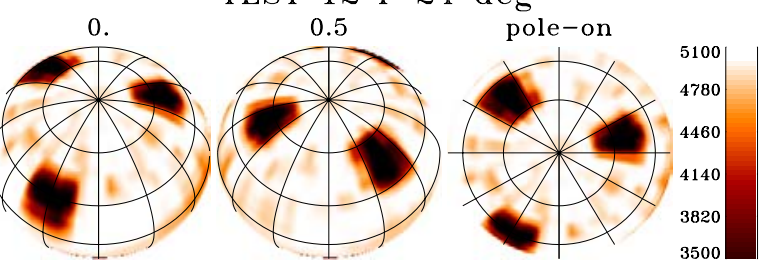

TEST $14 \mathrm{i}=36 \mathrm{deg}$
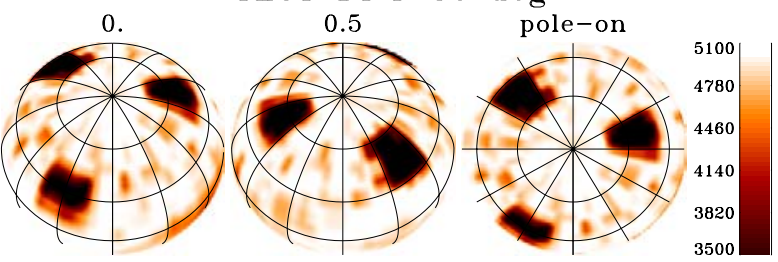

TEST $16 \mathrm{~T}=4850 \mathrm{~K}$
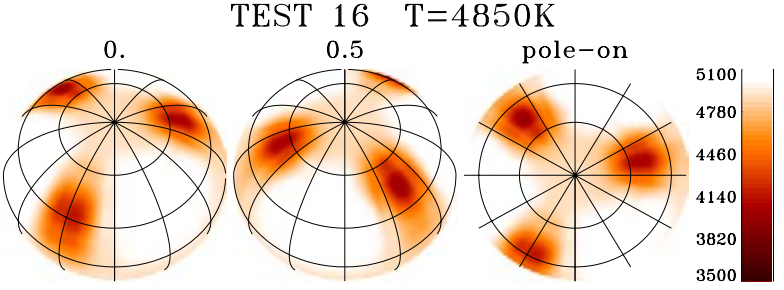

TEST $18 \mathrm{~T}=5150 \mathrm{~K}$
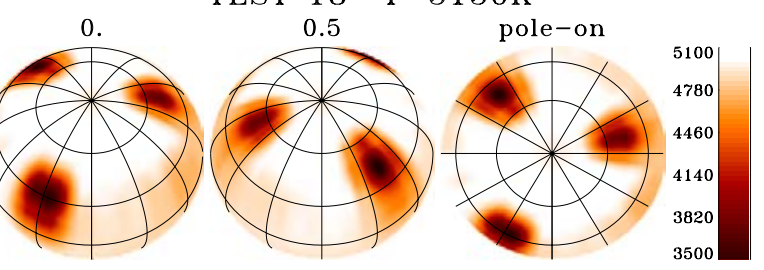

Fig. A.2. Tests 7-18: projected rotational velocity $v \sin i$; inclination $i$; and photospheric temperature $T$ (see Sect. A and Table A.1).

both models is possible but with higher surface "noise" in the final image in case $\mathrm{S} 2$ than for S1, and also with respect to the previous test \#20.

\section{A.5. Test 23-26: continuum misfits}

The effects of a systematic misfit of the continuum during the reduction procedure are shown in Fig. A.3, as was already demonstrated by Rice \& Strassmeier (2000) for the TEMPMAP code. The common definition of the continuum as a fit to the upper envelope of the absorption line spectrum is valid only for spectra that are not overcrowded with numerous atomic and molecular line features. For example for the spectra of the stars with $T_{\text {phot }}$ below $4750-4500 \mathrm{~K}$ no points of the actual continuum can be found for the spectral regions considered. Severe blending as well as other effects like convolution with 
I. S. Savanov and K. G. Strassmeier: Surface imaging using atomic and molecular features. I., Online Material p 5
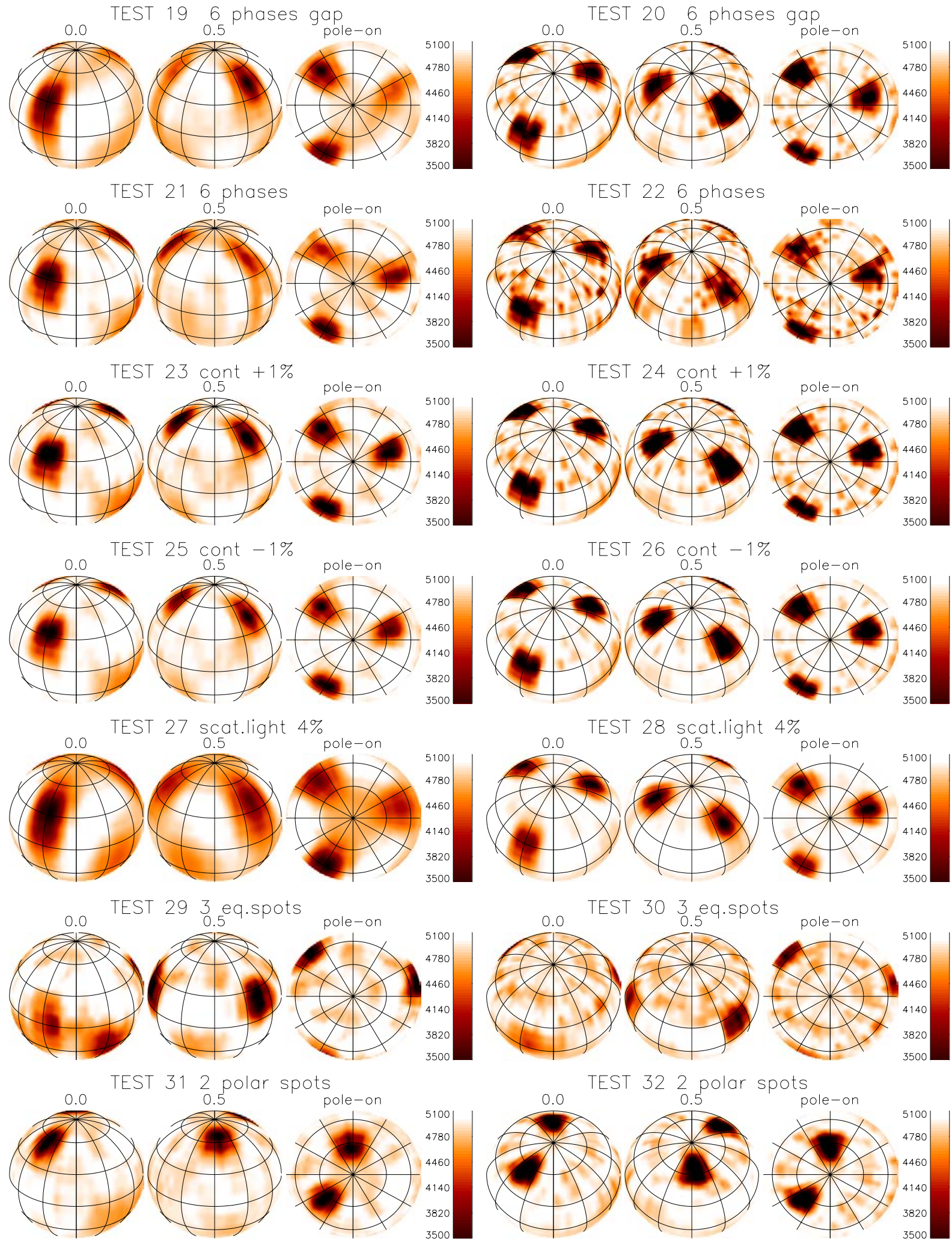

Fig. A.3. Tests 19-32: phase gap of 6 phases; just 6 out of 20 phases; continuum misplacement $\pm 1 \%$; $4 \%$ scattered light; three equatorial spots; and two high-latitude "polar" spots (see Sect. A and Table A.1).

instrumental profile, rotational broadening, etc. leads to an underestimation of the true continuum level. Another source of a possible systematic misfit of the continuum is related to the reduction procedures itself. The various astronomical data reduction packages (e.g. IRAF, MIDAS a.o.) offer different ways for continuum fitting both for the single-order coudé spectra and two-dimensional echelle spectra. 
I. S. Savanov and K. G. Strassmeier: Surface imaging using atomic and molecular features. I., Online Material p 6
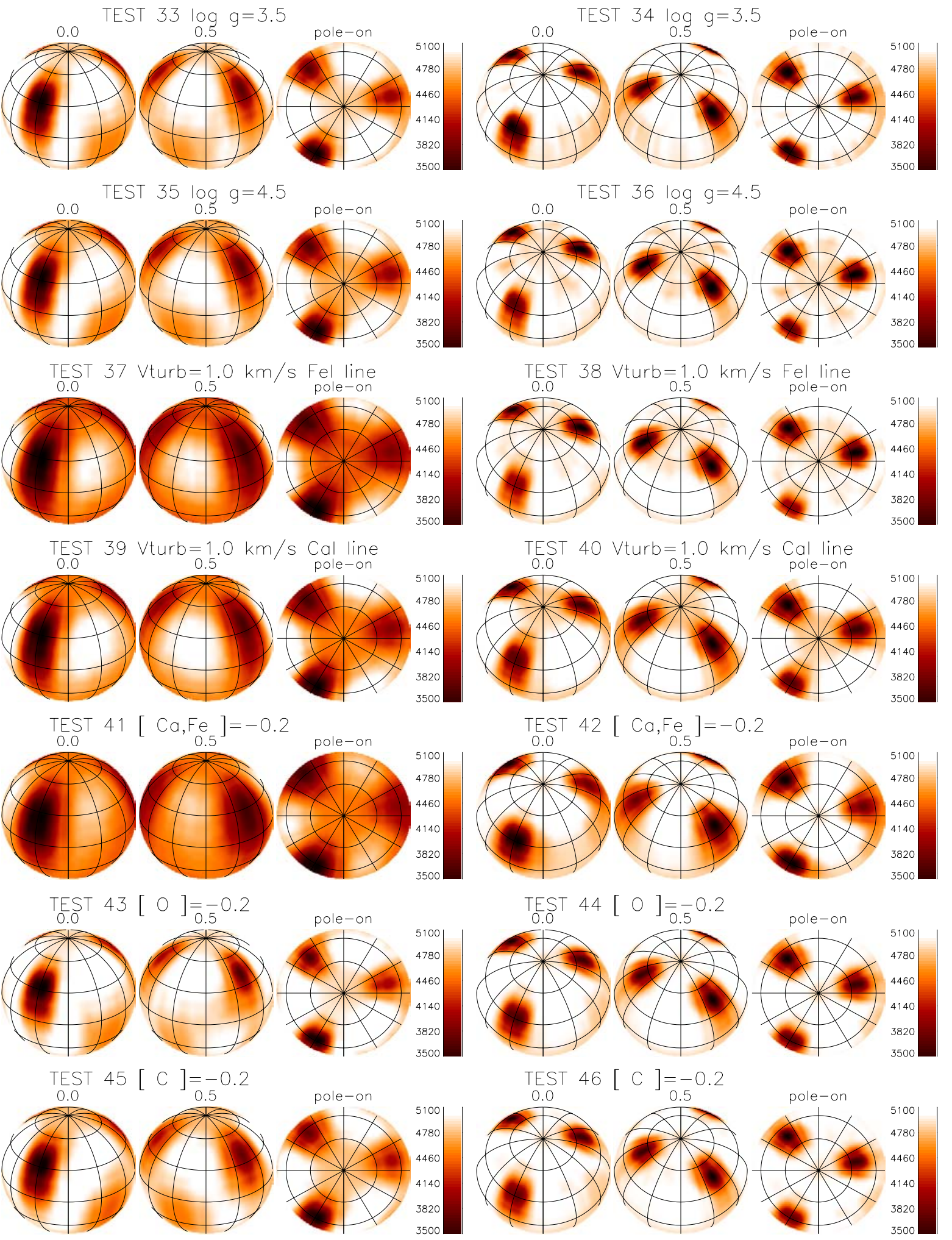

Fig. A.4. Tests 33-46: gravity $\log g$; microturbulence $V_{\text {turb }}$ for Fe I $6393 \AA$ and Ca I $6439 \AA$; change of Fe and Ca abundance [Ca, Fe]; and change of $\mathrm{O}$ and $\mathrm{C}$ abundance $[\mathrm{O}][\mathrm{C}]$ (see Sect. A and Table A.1 for details).

Our two test cases were reproduced with an artificial continuum offset of $\pm 1 \%$ (the positive value corresponds to higher continuum level and deeper line depths). We find that a reasonably correct recovery of the images can be obtained for both cases although in the case of positive offset the average temperature of the map is slightly lower by $150-200 \mathrm{~K}$ and vice versa for the negative offset. This is in agreement with the findings of Rice \& Strassmeier (2000) for TEMPMAP, despite the much more complicated surface structure of their artificial star. 
Table A.1. Summary of tests. The running number, \#, identifies the Test\#. "S" refers to the star number in Table 1. "Parameter" identifies the input parameter that was varied with respect to Table 1 and gives its adopted value. The $\sigma(x)$ values are the reconstructed temperature errors and their deviations along longitude at three different latitudinal bands (1: $+81^{\circ}, 2$ : sub-observer point, $3:-51^{\circ}$ for $\mathrm{S} 1$ and $-21^{\circ}$ for $\left.\mathrm{S} 2\right)$.

\begin{tabular}{|c|c|c|c|c|c|}
\hline$\#$ & $\mathrm{~S}$ & Parameter & $\sigma(x)_{1}$ & $\sigma(x)_{2}$ & $\sigma(x)_{3}$ \\
\hline 1 & $\mathrm{~S} 1$ & $S / N=200$ & $447 \pm 62$ & $58 \pm 8$ & $1045 \pm 165$ \\
\hline 2 & $\mathrm{~S} 1$ & $S / N=500$ & $83 \pm 12$ & $9 \pm 2$ & $396 \pm 37$ \\
\hline 3 & $\mathrm{~S} 1$ & $S / N=60$ & $1501 \pm 235$ & $582 \pm 90$ & $\geq 2000$ \\
\hline 4 & $\mathrm{~S} 2$ & $S / N=200$ & $298 \pm 43$ & $56 \pm 9$ & $915 \pm 132$ \\
\hline 5 & $\mathrm{~S} 2$ & $S / N=500$ & $37 \pm 5$ & $12 \pm 2$ & $139 \pm 20$ \\
\hline 6 & $\mathrm{~S} 2$ & $S / N=60$ & $762 \pm 124$ & $392 \pm 59$ & $\geq 2000$ \\
\hline 7 & $\mathrm{~S} 1$ & $v \sin i=22.0$ & $877 \pm 135$ & $98 \pm 15$ & $880 \pm 145$ \\
\hline 8 & $\mathrm{~S} 2$ & $v \sin i=48.0$ & $323 \pm 46$ & $63 \pm 10$ & $1076 \pm 148$ \\
\hline 9 & $\mathrm{~S} 1$ & $v \sin i=26.0$ & $464 \pm 66$ & $65 \pm 9$ & $866 \pm 145$ \\
\hline 10 & $\mathrm{~S} 2$ & $v \sin i=52.0$ & $292 \pm 42$ & $73 \pm 7$ & $947 \pm 132$ \\
\hline 11 & $\mathrm{~S} 1$ & $i=48^{\circ}$ & $347 \pm 52$ & $68 \pm 10$ & $1029 \pm 161$ \\
\hline 12 & $\mathrm{~S} 2$ & $i=24^{\circ}$ & $378 \pm 59$ & $79 \pm 12$ & $970 \pm 150$ \\
\hline 13 & $\mathrm{~S} 1$ & $i=72^{\circ}$ & $998 \pm 134$ & $57 \pm 9$ & $683 \pm 101$ \\
\hline 14 & $\mathrm{~S} 2$ & $i=36^{\circ}$ & $332 \pm 50$ & $59 \pm 9$ & $934 \pm 132$ \\
\hline 15 & $\mathrm{~S} 1$ & $T_{\mathrm{ph}}=4850$ & $774 \pm 114$ & $82 \pm 12$ & $936 \pm 157$ \\
\hline 16 & $\mathrm{~S} 2$ & $T_{\mathrm{ph}}=4850$ & $238 \pm 35$ & $40 \pm 6$ & $933 \pm 127$ \\
\hline 17 & $\mathrm{~S} 1$ & $T_{\mathrm{ph}}=5150$ & $514 \pm 75$ & $65 \pm 10$ & $960 \pm 161$ \\
\hline 18 & $\mathrm{~S} 2$ & $T_{\mathrm{ph}}=5150$ & $318 \pm 48$ & $52 \pm 8$ & $1037 \pm 152$ \\
\hline 19 & $\mathrm{~S} 1$ & 6 phase gap & $1005 \pm 161$ & $326 \pm 58$ & $\geq 2000$ \\
\hline 20 & $\mathrm{~S} 2$ & 6 phase gap & $694 \pm 106$ & $165 \pm 26$ & $\geq 2000$ \\
\hline 21 & $\mathrm{~S} 1$ & 6 phase & $973 \pm 161$ & $376 \pm 56$ & $\geq 2000$ \\
\hline 22 & $\mathrm{~S} 2$ & 6 phase & $793 \pm 126$ & $309 \pm 50$ & $\geq 2000$ \\
\hline 23 & $\mathrm{~S} 1$ & cont. $+1 \%$ & $671 \pm 97$ & $83 \pm 10$ & $793 \pm 132$ \\
\hline 24 & $\mathrm{~S} 2$ & cont. $+1 \%$ & $391 \pm 62$ & $64 \pm 9$ & $1080 \pm 157$ \\
\hline 25 & $\mathrm{~S} 1$ & cont. $-1 \%$ & $715 \pm 109$ & $93 \pm 12$ & $961 \pm 154$ \\
\hline 26 & $\mathrm{~S} 2$ & cont. $-1 \%$ & $340 \pm 51$ & $64 \pm 10$ & $1286 \pm 185$ \\
\hline 27 & $\mathrm{~S} 1$ & scat. $4 \%$ & $614 \pm 89$ & $67 \pm 10$ & $919 \pm 149$ \\
\hline 28 & $\mathrm{~S} 2$ & scat. $4 \%$ & $243 \pm 36$ & $36 \pm 5$ & $894 \pm 123$ \\
\hline 29 & $\mathrm{~S} 1$ & 3 eq. spots & $572 \pm 83$ & $79 \pm 12$ & $955 \pm 161$ \\
\hline 30 & $\mathrm{~S} 2$ & 3 eq. spots & $435 \pm 73$ & $52 \pm 8$ & $1041 \pm 152$ \\
\hline 31 & $\mathrm{~S} 1$ & 2 pol. spots & $615 \pm 89$ & $75 \pm 11$ & $918 \pm 160$ \\
\hline 32 & $\mathrm{~S} 2$ & 2 pol. spots & $296 \pm 46$ & $40 \pm 6$ & $897 \pm 120$ \\
\hline 33 & $\mathrm{~S} 1$ & $\log g=3.5$ & $690 \pm 102$ & $81 \pm 12$ & $1024 \pm 157$ \\
\hline 34 & $\mathrm{~S} 2$ & $\log g=3.5$ & $323 \pm 47$ & $39 \pm 6$ & $1010 \pm 147$ \\
\hline 35 & $\mathrm{~S} 1$ & $\log g=4.5$ & $798 \pm 116$ & $84 \pm 12$ & $911 \pm 149$ \\
\hline 36 & $\mathrm{~S} 2$ & $\log g=4.5$ & $369 \pm 55$ & $47 \pm 7$ & $1112 \pm 164$ \\
\hline 37 & $\mathrm{~S} 1$ & $\xi_{t}=1.06394$ & $657 \pm 95$ & $75 \pm 11$ & $918 \pm 154$ \\
\hline 38 & $\mathrm{~S} 2$ & $\xi_{t}=1.06394$ & $342 \pm 49$ & $48 \pm 7$ & $937 \pm 133$ \\
\hline 39 & $\mathrm{~S} 1$ & $\xi_{t}=1.06439$ & $800 \pm 115$ & $97 \pm 14$ & $970 \pm 163$ \\
\hline 40 & $\mathrm{~S} 2$ & $\xi_{t}=1.06439$ & $318 \pm 47$ & $40 \pm 6$ & $1152 \pm 164$ \\
\hline 41 & $\mathrm{~S} 1$ & {$[\mathrm{Ca}, \mathrm{Fe}]=-0.2$} & $945 \pm 145$ & $92 \pm 17$ & $1036 \pm 169$ \\
\hline 42 & $\mathrm{~S} 2$ & {$[\mathrm{Ca}, \mathrm{Fe}]=-0.2$} & $279 \pm 39$ & $32 \pm 5$ & $771 \pm 111$ \\
\hline 43 & $\mathrm{~S} 1$ & {$[\mathrm{O}]=-0.2$} & $654 \pm 98$ & $77 \pm 12$ & $856 \pm 141$ \\
\hline 44 & $\mathrm{~S} 2$ & {$[\mathrm{O}]=-0.2$} & $365 \pm 55$ & $53 \pm 8$ & $900 \pm 128$ \\
\hline 45 & $\mathrm{~S} 1$ & {$[C]=-0.2$} & $578 \pm 177$ & $69 \pm 10$ & $926 \pm 150$ \\
\hline 46 & $\mathrm{~S} 2$ & {$[C]=-0.2$} & $354 \pm 52$ & $48 \pm 7$ & $851 \pm 121$ \\
\hline
\end{tabular}

\section{A.6. Test 27-28: scattered light}

Also following Rice \& Strassmeier (2000), we attempt to simulate the presence of scattered light by adding a value of $4 \%$ to all data (Fig. A.3). Other parameters were assumed to be known precisely and a $S / N$ ratio of 200:1 was adopted. Even for this unrealistically large value of the scattering light the position of the spots was restored correctly for both test cases but their contrast appeared reduced by 200-300 K. For the highinclination case $\mathrm{S} 1$, the spots additionally became elongated in the latitudinal direction.

\section{A.7. Test 29-32: latitude resolution}

Among the common intrinsic limits of all Doppler imaging codes is the ambiguity between the hemispheres once the inclination is near $70^{\circ}-90^{\circ}$. A lack of latitude resolution near the low latitude regions is expected and, according to our estimations, this is the region with the largest errors. We have created two special test cases. First the input image consisted of the usual three spots but placed only along the equator at (central) latitudes of $-15^{\circ},+15^{\circ}$ and $0^{\circ}$ (tests 29-30) and, second, where the input images consisted of only two high-latitude spots centered at latitudes of $+60^{\circ}$ and $+54^{\circ}$ (tests $31-32$ ). The quasi symmetric high-latitude spots, a typical detail often found in real Doppler imaging applications, are restored with high accuracy, even in shape. The low-latitude spots are only partly recovered. The high-inclination case S1 misplaced one of the three spots to lower latitudes by a few degrees, while the lowinclination case $\mathrm{S} 2$ led, as expected, to the better result.

\section{A.8. Test 33-40: gravity and microturbulence}

Local line profiles are strongly dependent on the atomic and model input parameters. The sensitivity of the theoretical spectra to changes in these parameters is well established from numerous spectroscopic investigations (e.g. Gray 1992). It is well known that, in general, the formation of lines of neutral elements is sensitive to changes in effective temperature while lines of ionized elements are sensitive to changes in gravity. Weak lines are practically insensitive to changes of, what we still call, the parameter of microturbulence while the equivalent width of strong lines should vary significantly with microturbulence. Changes of the transition probabilities ( $\log g f$ values) are linearly proportional to the changes of abundances (see, again, Rice \& Strassmeier 2000). Detailed treatment of the radiative transfer problem leads to the conclusion that each of these relations is valid within certain ranges of temperatures and gravities or, equivalently, to limited ranges of these parameters. For example, the strength of a line is proportional to the abundance in a curve-of-growth style and shows linear changes only for the weak lines.

The influence of uncertainties of this kind on our image restoration problem can only be understood if we supplement it by the analysis of changes in the local line profiles. This is shown in Fig. 1. In this figure, initial synthetic spectra are shown by thin lines while thick lines represent the calculated line profiles with some specific parameter changed. 
The parameters are gravity, microturbulence, $\mathrm{Ca}$ and $\mathrm{Fe}$ abundance and $\mathrm{O}$ abundance. We compare calculations in two wavelength regions containing Fe I 6411 A (left column in Fig. 1) and Ca I $6439 \AA$ (right column), that belong to the lines widely used for image reconstruction. The nominal synthetic spectra are always calculated for 11 values of $T_{\text {phot }}$, from 3500 to $6000 \mathrm{~K}$ in steps of $250 \mathrm{~K}$, zero microturbulence, the classical values for the damping constants and convolved with an instrumental profile corresponding to a resolution of $\Delta \lambda / \lambda=60000$. Gravity $\log g$ was adopted to be 3.5 .

The figure demonstrates the influence of molecular intensities (mainly $\mathrm{TiO}$ ) for temperatures below $4500-4250 \mathrm{~K}$. The first row in Fig. 1 illustrates the sensitivity to changes of the surface gravity $(\Delta \log g=0.5)$ although both lines, Fe I $6411 \AA$ and the Ca I $6439 \AA$, are neutral and should mainly reflect only changes in temperature. In case of the Ca I 6439-Å line, which is stronger than the $\mathrm{Fe} \mathrm{I}$ line and is formed in a saturated regime of the curve-of-growth, we can notice a broadening of its line profile for the temperatures between 4000-4500 K, typical spot temperatures. Note though that the non-monotonic behavior of the combined changes of the $\mathrm{Ca}$ I line and the $\mathrm{TiO}$ band intensities is due to the gravity change.

The second row in Fig. 1 shows the intensity variations due to the changes in microturbulent velocity from $0 \mathrm{~km} \mathrm{~s}^{-1}$ to $2 \mathrm{~km} \mathrm{~s}^{-1}$. For the weak lines the changes are negligible while, as the lines become stronger, the influence on its intensities grows (as expected).

Finally, the tests 33-36 in Fig. A.4 show the influences of the parameter $\log g$ for the image reconstruction. The values listed in Table 1 were used for the forward calculations. Input atmospheres were taken from grids of models with $\log g=4.5$ and 3.5 instead of 4.0 for the forward calculations. The result is that the photospheric temperature is lowered most at and near the rotational pole for the overestimated case (4.5) while it is lowered most at or near the equator for the underestimated case $(\log g=3.5)$. The code copes with this redistribution problem slightly differently for the high-inclination and the lowinclination cases (Fig. A.4).

The forward calculations were made with the microturbulence parameter $\xi_{t}$ set to $0.0 \mathrm{~km} \mathrm{~s}^{-1}$ while the tests $37-40$ assumed $\xi_{t}=1.0 \mathrm{~km} \mathrm{~s}^{-1}$. As well as the often-used Dopplerimaging line Ca I $6439 \AA$ in tests 39 and 40 in Fig. A.4, the Fe I line at $6393 \AA$ was used for tests 37 and 38. The latter line was chosen because we would like to perform a reconstruction from two lines with approximately similar sensitivity to certain parameters but from different chemical species.
The high-inclination case S1 turned out to be more sensitive to the artificial microturbulence than the low-inclination case, independent of the spectral line. Although the spot locations are correctly reproduced, their size and shape is significantly blurred (tests 37 and 39). The average surface temperature is underestimated by $\Delta T \approx 300 \mathrm{~K}$. The low-inclination case $\mathrm{S} 2$ is comparably less sensitive to micoturbulence although its average temperature is overestimated by $\Delta T \approx 300 \mathrm{~K}$. Here, the Fe I line shows a better behavior than the Ca I line.

\section{A.9. Test 41-46: abundances of $\mathrm{Fe}, \mathrm{Ca}, \mathrm{O}$ and $\mathrm{C}$}

The last tests are related to the changes of abundances. Several common features can characterize the results from these tests. For the high-inclination S1 cases, the restorations result in an overall cooler surface as well as in an enlargement of spots in the latitude direction together with a poorer reconstruction of the equatorial spot compared to the high-latitude spots. For the low-inclination S2 cases, the restorations result in a reduction of size and contrast of the spots.

All our test were performed with solar abundances as input for the forward calculations. Synthetic spectra were computed with the abundances of $\mathrm{Ca}$ and $\mathrm{Fe}$ reduced by 0.2 dex relative to the solar values ${ }^{2}$. These spectra are shown for 11 temperatures in Fig. 1c. Note again the differences in the behavior of the lines. The Fe I 6411- $\AA$ line, which is on the so-called flat part of the curve-of-growth, is practically unchanging in strength, while the strongly saturated Ca I $6439-\AA ̊$ line is changing the broad damping wings.

The last two panels in Fig. 1 show theoretical spectra computed with an oxygen abundance reduced by 0.2 dex relative to the solar value. Models with effective temperatures below $\approx 4250 \mathrm{~K}$ strongly depend on the correct oxygen abundance due to the changes in $\mathrm{TiO}$ molecular intensities. Therefore, an incorrect oxygen abundance can influence the restoration for the cases with spots of temperatures below $4250 \mathrm{~K}$. Although no atomic lines of elements like $\mathrm{C}$ and $\mathrm{N}$ are present in the spectral region $6400-6440 \AA$, we noticed the importance of having a good estimate for the abundances of the $\mathrm{CNO}$ elements (which also characterize the evolutionary status of the investigated object). This is because a change in carbon abundance influences the molecular equilibrium calculations via $\mathrm{CO}$ molecular formation. At the same time many spectral regions are strongly contaminated by $\mathrm{CN}$ absorption (e.g. the often-used Li I 6708-Å region).

\footnotetext{
2 Abundances $(A)$ expressed in brackets are relative to solar values; $[A]=\log \varepsilon(A)-\log \varepsilon(A)_{\odot}$ with $\log \varepsilon(A)=\log (A / \mathrm{H})+12.0$.
} 